\title{
On the global well-posedness of a class of Boussinesq-Navier-Stokes systems
}

\author{
Changxing Miao and Liutang Xue
}

\begin{abstract}
In this paper we consider the following 2D BoussinesqNavier-Stokes systems

$$
\begin{aligned}
\partial_{t} u+u \cdot \nabla u+\nabla p & =-\nu|D|^{\alpha} u+\theta e_{2} \\
\partial_{t} \theta+u \cdot \nabla \theta & =-\kappa|D|^{\beta} \theta \\
\operatorname{div} u & =0
\end{aligned}
$$

with $\nu>0, \kappa>0$ and $0<\beta<\alpha<1$. When $\frac{6-\sqrt{6}}{4}(\doteq 0.888)<\alpha<1$, $1-\alpha<\beta \leq f(\alpha)$, where $f(\alpha)<1$ is an explicit function as a technical bound, we prove the global well-posedness results for the rough initial data.
\end{abstract}

Mathematics Subject Classification (2000). 76D03, 76D05, 35B33, 35Q35.

Keywords. Boussinesq system, Regularization effect, Para-differential calculus, Global well-posedness.

\section{Introduction}

The 2D generalized Boussinesq systems are of the forms

$$
\left\{\begin{array}{l}
\partial_{t} u+u \cdot \nabla u+\nabla p+\nu|D|^{\alpha} u=\theta e_{2}, \quad(t, x) \in \mathbb{R}^{+} \times \mathbb{R}^{2} \\
\partial_{t} \theta+u \cdot \nabla \theta+\kappa|D|^{\beta} \theta=0 \\
\operatorname{div} u=0 \\
\left.u\right|_{t=0}=u^{0},\left.\quad \theta\right|_{t=0}=\theta^{0},
\end{array}\right.
$$

where $\nu \geq 0, \kappa \geq 0, e_{2}=(0,1)$ the canonical vector, $(\alpha, \beta) \in[0,2]^{2}$ and the fractional differential operator $|D|^{\alpha}$ is defined via the Fourier transform

$$
|D|^{\alpha} f(x)=\frac{1}{4 \pi^{2}} \int_{\mathbb{R}^{2}} e^{i x \cdot \xi}|\xi|^{\alpha} \mathcal{F}(f)(\xi) \mathrm{d} \xi .
$$

These systems are meaningful generalization of the simplified models widely used in the modeling of the oceanic and atmospheric motions (cf. [16]). Here, the divergence-free vector field $u=\left(u^{1}, u^{2}\right)$ denotes the velocity, scalar functions $\theta, p$ denote the temperature and the pressure respectively, the absolute 
constants $\nu, \kappa$ can be seen as the inverse of the Reynolds numbers. The term $\theta e_{2}$ in the velocity equation models the effect of gravity on the fluid motion. If $\theta^{0}=0$, from the maximum principle of the transport-diffusion equation (i.e. Proposition 2.2 below), the systems are naturally reduced to the $2 \mathrm{D}$ generalized Navier-Stokes(Euler) equations.

From a mathematical view, the fully viscous model with $\nu>0, \kappa>0, \alpha=$ $\beta=2$ is the simplest one to study. It acts very similar to the $2 \mathrm{D}$ Navier-Stokes equation and similar global results can be achieved. On the other hand, the most difficult one for the mathematical study is the inviscid model, that is when $\nu=\kappa=0$. Up to now, only local existence results can be proven.

Here we focus on the cases where the dissipation effect in the velocity equation plays a dominant role. The most typical models are those with the diffusion effect in the temperature equation neglected $(\kappa=0, \nu>0)$, and there have been some recent important works on these Boussinesq systems. For the case with the full viscosity, i.e. when $\alpha=2$, global well-posedness results can be established in various functional spaces. In $[3,13]$, the authors independently proved that for large initial data $\left(u^{0}, \theta^{0}\right) \in H^{s} \times H^{s}$ with $s>2$ the system is global well-posed. Later on, Hmidi and Keraani in [9] showed global well-posedness for less regular data $\left(u^{0}, \theta^{0}\right) \in H^{s} \times H^{s}$ with $s>0$. In [8], Danchin and Paicu proved the unconditional uniqueness in the energy space $L^{2} \times L^{2}$. For the cases with weaker dissipation, i.e. when $1 \leq \alpha<2$, the problem is also solvable. When $\alpha \in] 1,2[$, as in [9] through taking advantage of the maximal regularity estimates for the semi-group $e^{-t|D|^{\alpha}}$, one can prove the global well-posedness (see also [18]). For the subtle critical case $\alpha=1$, Hmidi et al. in [12] proved the global result for the rough data through exploiting the new structural properties.

If we further weaken the viscosity effect in the velocity equation to the $\alpha<1$ case, then to obtain the global strong solutions, it seems that introducing the viscosity effect in the temperature equation $(\kappa>0)$ is necessary and meanwhile $\beta$ should satisfy $\beta \geq 1-\alpha$. In fact, we have a rough observation from the coupled system of temperature $\theta$ and vorticity $\omega$, where $\omega$ is defined by $\omega:=\operatorname{curl} u=\partial_{1} u^{2}-\partial_{2} u^{1}$. The coupled system writes

$$
\left\{\begin{array}{l}
\partial_{t} \omega+u \cdot \nabla \omega+\nu|D|^{\alpha} \omega=\partial_{1} \theta, \\
\partial_{t} \theta+u \cdot \nabla \theta+\kappa|D|^{\beta} \theta=0, \\
\left.\omega\right|_{t=0}=\omega^{0}:=\operatorname{curl}^{0},\left.\quad \theta\right|_{t=0}=\theta^{0} .
\end{array}\right.
$$

As the known Beale-Kato-Majda blowup criterion (cf. [2]) shows, the global continuation of the system (1.1) is closely related to the a priori bound of $\|\omega\|_{L_{t}^{1} L^{\infty}}$. Thus to get the key uniform estimates on $\omega$, the maximal gain of $\alpha$ derivative from the dissipation term should at least roughly compensate the loss of one derivative in $\theta$ in the vorticity equation with the help of the diffusion effect in the temperature equation, from which at most $\beta$ derivative in $\theta$ is gained. Hence $\alpha+\beta \geq 1$ is needed. This is also the sense in which the case $\{\alpha=1, \nu>0, \kappa=0\}$ is called a critical case. 
In this paper we study the model when $\nu>0, \kappa>0$ and $0<\beta<\alpha<1$. For brevity, we always set $\nu=\kappa=1$ in the following. We shall adopt the subtle method introduced in $[11,12]$ to study the delicate coupled effects of these generalized cases. More precisely, our main result is the following

Theorem 1.1. Let $\left.(\alpha, \beta) \in \Pi:=] \frac{6-\sqrt{6}}{4}, 1[\times] 1-\alpha, f(\alpha)\right]$ (for $\Pi$ see Fig. 1 in the sequel) with $f(\alpha):=\min \left\{\frac{7+2 \sqrt{6}}{5} \alpha-2, \frac{\alpha(1-\alpha)}{\sqrt{6}-2 \alpha}, 2-2 \alpha\right\}$. Assume that $\theta^{0} \in$ $H^{1-\alpha} \cap B_{\infty, 1}^{1-\alpha}$ and $u^{0}$ is a divergence-free vector field belonging to $H^{1} \cap \dot{W}^{1, p}$ with $p \in] \frac{2}{\alpha+\beta-1}, \infty[$, then the system (1.1) has a unique global solution $(u, \theta)$ such that for every $\sigma \in\left[1, \frac{\alpha}{1-\alpha+2 / p}[\right.$,

$$
\begin{aligned}
& u \in \mathcal{C}\left(\mathbb{R}^{+}, H^{1}\right) \cap L^{\infty}\left(\mathbb{R}^{+}, \dot{W}^{1, p}\right) \cap \widetilde{L}_{l o c}^{\sigma}\left(\mathbb{R}^{+}, B_{\infty, 1}^{1}\right) \text { and } \\
& \theta \in \mathcal{C}\left(\mathbb{R}^{+}, H^{1-\alpha} \cap B_{\infty, 1}^{1-\alpha}\right) \cap \widetilde{L}_{\text {loc }}^{1}\left(\mathbb{R}^{+}, H^{1-\alpha+\beta} \cap B_{\infty, 1}^{1-\alpha+\beta}\right) .
\end{aligned}
$$

Besides, if $u^{0} \in H^{1} \cap \dot{B}_{p, 2}^{1}$, we can also prove $u \in \mathcal{C}\left(\mathbb{R}^{+}, H^{1} \cap \dot{B}_{p, 2}^{1}\right)$.

For the definitions of Besov spaces $B_{p, r}^{s}$ and the mixed space-time Besov spaces $\widetilde{L}_{T}^{\varrho} B_{p, r}^{s}$ see the next section below.

We now give some comments.

Remark 1.1. We note that the cases $(\alpha, \beta) \in \Xi:=] 0,1\left[{ }^{2} \cap\{\alpha+\beta \geq 1\}\right.$ are definitely nontrivial cases, and it seems mathematically hard to deal with these models, even for the sub-critical range. In fact, to obtain the global wellposedness of (1.1), all the past works treated the cases needing $\alpha \geq 1$ or $\beta \geq 1$, and the regularization effects from the dominant viscosity terms are strong enough so that it suffices to fully exploit these effects to overcome the loss of one derivative in $\theta$ in the vorticity equation (maybe except $[11,12]$ ). But this way fails for the cases $(\alpha, \beta) \in \Xi$, and one has to truly take advantage of the coupled regularization effects from both viscosity terms. It is a pity that till now there are not very effective methods to treat these cases: if one only relies on the standard energy method, then just as [17] shows, the condition $\alpha+\beta \geq 2$ is needed and can do nothing with the potential cases $\Xi$; On the other hand, as we show in this paper, the method of applying the new structures (in its current state) is workable but very restrictive.

Remark 1.2. In our cases $\Pi$, we need a complicated explicit function $f(\alpha)$ as an upper bound, and this is a technical assumption due to that we have to consider $|D|^{\beta-\alpha} \partial_{1} \theta$ as a forcing term (see below). But since it is commonly believed that the viscosity terms are always good terms and the larger the power $\alpha$ (or $\beta$ ) is, the better effects they produce, our results strongly suggest that all the cases $(\alpha, \beta) \in] \frac{6-\sqrt{6}}{4}, 1[\times] 1-\alpha, 1[$ should be globally well-posed. Of course, it still needs a further strict proof.

Remark 1.3. We notice that the assumption on the initial data is immediately satisfied if $\left(\theta^{0}, u^{0}\right) \in H^{s_{1}} \times\left[H^{s_{2}}\right]^{2}$ with $s_{1}>2-\alpha$ and $s_{2}>3-\alpha-\beta$.

The main idea in the proof of Theorem 1.1 is to use the structures of the system solved by $(\omega, \theta)$, which is motivated by $[11,12]$. To get a first glance, 
we shall neglect the nonlinear terms here, then the coupled system of $(\omega, \theta)$ reduces to

$$
\partial_{t} \omega+|D|^{\alpha} \omega=\partial_{1} \theta, \quad \partial_{t} \theta+|D|^{\beta} \theta=0
$$

Thus

$$
\partial_{t} \omega+|D|^{\alpha}\left(\omega-|D|^{-\alpha} \partial_{1} \theta\right)=0, \quad \partial_{t} \theta+|D|^{\beta} \theta=0 .
$$

Set $\mathcal{R}_{\alpha}:=|D|^{-\alpha} \partial_{1}$, then

$$
\partial_{t}\left(\omega-\mathcal{R}_{\alpha} \theta\right)+|D|^{\alpha}\left(\omega-\mathcal{R}_{\alpha} \theta\right)=|D|^{\beta-\alpha} \partial_{1} \theta, \quad \partial_{t} \theta+|D|^{\beta} \theta=0 .
$$

If roughly $\alpha \sim 1, \beta \sim 0$, the forcing term $|D|^{\beta-\alpha} \partial_{1} \theta$ has much less loss of derivatives than term $\partial_{1} \theta$ and indeed we have some good estimates on $\omega-\mathcal{R}_{\alpha} \theta$. These estimates will strongly help to obtain the important estimates on $\omega$.

To prove Theorem 1.1, we shall use the same idea: we shall study the new equation to get a priori estimates on $\omega-\mathcal{R}_{\alpha} \theta$ and then return to obtain the crucial estimates on $\omega$. During this process, some difficulty will be encountered. The first one is to estimate the commutator $\left[\mathcal{R}_{\alpha}, u \cdot \nabla\right]$ which naturally turns up when the nonlinear term is taken into account; the second is to derive the $L^{p}$ estimates on the new unknown quantity $\omega-\mathcal{R}_{\alpha} \theta$; another one is to obtain estimates on $\omega$ from estimates on $\omega-\mathcal{R}_{\alpha} \theta$ (since in contrast with the Riesz transform, $\mathcal{R}_{\alpha}$ is not $L^{p}$-bounded and roughly contains positive derivative of $1-\alpha$ power). We shall treat such commutator estimates in Sect. 3, and carefully consider the range of $(\alpha, \beta)$ to tackle the second difficulty, and yet we shall sufficiently apply the regularization effect (Proposition 2.3 below) of the temperature equation to overcome the third one.

The paper is organized as follows. Section 2 is devoted to present some preparatory results on Besov spaces. Some estimates about linear transportdiffusion equation are also given. In Sect. 3, commutator estimates involving $\mathcal{R}_{\alpha}$ are studied. Section 4 is the main part dedicated to the proof of Theorem 1.1. Finally, some technical lemmas are shown in Sect. 5.

\section{Preliminaries}

\subsection{Notations}

Throughout this paper the following notations will be used.

$\diamond$ The notation $X \lesssim Y$ means that there exists a positive harmless constant $C$ such that $X \leq C Y . X \approx Y$ means that both $X \lesssim Y$ and $Y \lesssim X$ are satisfied.

$\diamond \mathcal{S}$ denotes the Schwartz class, $\mathcal{S}^{\prime}$ the space of tempered distributions, and $\mathcal{S}^{\prime} / \mathcal{P}$ the quotient space of tempered distributions up to polynomials.

$\diamond$ We use $\mathcal{F} f$ or $\widehat{f}$ to denote the Fourier transform of a tempered distribution $f$.

$\diamond$ For any pair of operators $A$ and $B$ on some Banach space $\mathcal{X}$, the commutator $[A, B]$ is defined by $A B-B A$. 
$\diamond$ For every $k \in \mathbb{Z}^{+}$, the notion $\Phi_{k}$ denotes any function of the form

$$
\Phi_{k}(t)=C_{0} \underbrace{\exp (\ldots \exp }_{k \text { times }}\left(C_{0} t\right) \ldots)
$$

where $C_{0}$ depends on the related norms of the initial data and its value may be different from line to line up to some absolute constants.

\subsection{Littlewood-Paley decomposition and Besov spaces}

To define Besov space we need the following dyadic partition of unity (cf. [4]). Choose two nonnegative radial functions $\chi, \varphi \in C^{\infty}\left(\mathbb{R}^{n}\right)$ be supported respectively in the ball $\left\{\xi \in \mathbb{R}^{n}:|\xi| \leq \frac{4}{3}\right\}$ and the shell $\left\{\xi \in \mathbb{R}^{n}: \frac{3}{4} \leq|\xi| \leq \frac{8}{3}\right\}$ such that

$$
\chi(\xi)+\sum_{j \geq 0} \varphi\left(2^{-q} \xi\right)=1, \quad \forall \xi \in \mathbb{R}^{n} ; \quad \sum_{q \in \mathbb{Z}} \varphi\left(2^{-q} \xi\right)=1, \quad \forall \xi \neq 0 .
$$

For all $f \in \mathcal{S}^{\prime}\left(\mathbb{R}^{n}\right)$ we define the nonhomogeneous Littlewood-Paley operators

$$
\Delta_{-1} f:=\chi(D) f ; \forall q \in \mathbb{N} \quad \Delta_{q} f:=\varphi\left(2^{-q} D\right) f \quad \text { and } \quad S_{q} f:=\sum_{-1 \leq j \leq q-1} \Delta_{j} f .
$$

The homogeneous Littlewood-Paley operators are defined as follows

$$
\forall q \in \mathbb{Z}, \quad \dot{\Delta}_{q} f:=\varphi\left(2^{-q} D\right) f, \quad \dot{S}_{q} f:=\sum_{j \leq q-1} \dot{\Delta}_{j} f .
$$

The paraproduct between two distributions $f$ and $g$ is defined by

$$
T_{f} g:=\sum_{q \in \mathbb{N}} S_{q-1} f \Delta_{q} g .
$$

Thus we have the following formal decomposition known as Bony's decomposition

$$
f g=T_{f} g+T_{g} f+R(f, g)
$$

where

$$
R(f, g):=\sum_{q \geq-1} \Delta_{q} f \widetilde{\Delta}_{q} g, \quad \text { and } \quad \widetilde{\Delta}_{q}:=\Delta_{q-1}+\Delta_{q}+\Delta_{q+1} .
$$

Now we introduce the definition of Besov spaces. Let $(p, r) \in[1, \infty]^{2}, s \in \mathbb{R}$, the nonhomogeneous Besov space $B_{p, r}^{s}$ is defined as the set of tempered distribution $f$ such that

$$
\|f\|_{B_{p, r}^{s}}:=\left\|\left\{2^{q s}\left\|\Delta_{q} f\right\|_{L^{p}}\right\}_{q \geq-1}\right\|_{\ell^{r}}<\infty
$$

The homogeneous space $\dot{B}_{p, r}^{s}$ is the set of $f \in \mathcal{S}^{\prime}\left(\mathbb{R}^{n}\right) / \mathcal{P}\left(\mathbb{R}^{n}\right)$ such that

$$
\|f\|_{\dot{B}_{p, r}^{s}}:=\left\|\left\{2^{q s}\left\|\dot{\Delta}_{q} f\right\|_{L^{p}}\right\}_{q \in \mathbb{Z}}\right\|_{\ell^{r}(\mathbb{Z})}<\infty .
$$

We point out that for all $s \in \mathbb{R}, B_{2,2}^{s}=H^{s}$ and $\dot{B}_{2,2}^{s}=\dot{H}^{s}$.

Next we introduce two kinds of coupled space-time Besov spaces. The first one $L^{\varrho}\left([0, T], B_{p, r}^{s}\right)$, abbreviated by $L_{T}^{\varrho} B_{p, r}^{s}$, is the set of tempered distribution $f$ such that

$$
\|f\|_{L_{T}^{\varrho} B_{p, r}^{s}}:=\|\|\left\{2^{q s}\left\|\Delta_{q} f\right\|_{L^{p}}\right\}_{q \geq-1}\left\|_{\ell^{r}}\right\|_{L_{T}^{\varrho}}<\infty .
$$


The second one $\widetilde{L}^{\varrho}\left([0, T], B_{p, r}^{s}\right)$, abbreviated by $\widetilde{L}_{T}^{\varrho} B_{p, r}^{s}$, is the set of tempered distribution $f$ satisfying

$$
\|f\|_{\widetilde{L}_{T}^{\varrho} B_{p, r}^{s}}:=\left\|\left\{2^{q s}\left\|\Delta_{q} f\right\|_{L_{T}^{\varrho} L^{p}}\right\}_{q \geq-1}\right\|_{\ell^{r}}<\infty .
$$

Due to Minkowiski inequality, we immediately obtain

$$
L_{T}^{\varrho} B_{p, r}^{s} \hookrightarrow \widetilde{L}_{T}^{\varrho} B_{p, r}^{s} \text {, if } r \geq \rho \quad \text { and } \quad \widetilde{L}_{T}^{\varrho} B_{p, r}^{s} \hookrightarrow L_{T}^{\varrho} B_{p, r}^{s} \text {, if } \varrho \geq r \text {. }
$$

We can similarly extend to the homogeneous ones $L_{T}^{\varrho} \dot{B}_{p, r}^{s}$ and $\widetilde{L}_{T}^{\varrho} \dot{B}_{p, r}^{s}$. (cf. [4])

Berstein's inequality is fundamental in the analysis involving Besov spaces

Lemma 2.1. Let $f \in L^{a}, 1 \leq a \leq b \leq \infty$. Then for every $(k, q) \in \mathbb{N}^{2}$ there exists a constant $C>0$ such that

$$
\begin{gathered}
\sup _{|\alpha|=k}\left\|\partial^{\alpha} S_{q} f\right\|_{L^{b}} \leq C 2^{q\left(k+n\left(\frac{1}{a}-\frac{1}{b}\right)\right)}\|f\|_{L^{a}}, \\
C^{-1} 2^{q k}\|f\|_{L^{a}} \leq \sup _{|\alpha|=k}\left\|\partial^{\alpha} \Delta_{q} f\right\|_{L^{a}} \leq C 2^{q k}\|f\|_{L^{a}}
\end{gathered}
$$

\subsection{Transport-diffusion equation}

In this subsection we shall collect some useful estimates for the smooth solutions of the following linear transport-diffusion equation

$$
(T D)_{\beta} \quad\left\{\begin{array}{l}
\partial_{t} \theta+u \cdot \nabla \theta+|D|^{\beta} \theta=f, \quad \beta \in[0,1] \\
\operatorname{div} u=0,\left.\quad \theta\right|_{t=0}=\theta^{0} .
\end{array}\right.
$$

The $L^{p}$ estimate for $(\mathrm{TD})_{\beta}$ equation is shown in [6].

Proposition 2.2. Let $u$ be a smooth divergence-free vector field of $\mathbb{R}^{n}$ and $\theta$ be a smooth solution of $(T D)_{\beta}$. Then for every $p \in[1, \infty]$ we have

$$
\|\theta(t)\|_{L^{p}} \leq\left\|\theta_{0}\right\|_{L^{p}}+\int_{0}^{t}\|f(\tau)\|_{L^{p}} d \tau .
$$

The following smoothing effect plays a key role in the proof.

Proposition 2.3. Let $u$ be a smooth divergence-free vector field of $\mathbb{R}^{n}$ with vorticity $\omega$ and $\theta$ be a smooth solution of $(T D)_{\beta}$. Then for every $\left.(p, \varrho) \in\right] 1$, $\infty[\times[1, \infty]$ we have

$$
\sup _{q \in \mathbb{N}} 2^{q \frac{\beta}{\varrho}}\left\|\Delta_{q} \theta\right\|_{L_{t}^{\varrho} L^{p}} \lesssim \varrho, p\left\|\theta^{0}\right\|_{L^{p}}+\left\|\theta^{0}\right\|_{L^{\infty}}\|\omega\|_{L_{t}^{1} L^{p}}+\|f\|_{L_{t}^{1} L^{p}} .
$$

Remark 2.1. For $\varrho=1, \beta=1$ and $f=0$, the result has appeared in [11]. Here with necessary modifications, this generalized case can be treated in a similar way.

We also have the classical regularization effects as follows (cf. [10,15]).

Proposition 2.4. Let $-1<s<1,\left(\varrho, \varrho_{1}, p, r\right) \in[1, \infty]^{4}, \varrho_{1} \leq \varrho$ and $u$ be a divergence-free vector field belonging to $L_{\text {loc }}^{1}\left(\mathbb{R}^{+} ; \operatorname{Lip}\left(\mathbb{R}^{n}\right)\right)$. We consider a smooth solution $\theta$ of the equation $(T D)_{\beta}$, then there exists $C>0$ such that for every $t \in \mathbb{R}^{+}$,

$$
\|\theta\|_{\widetilde{L}_{t}^{\infty} B_{p, r}^{s}}+\left\|\left(\mathrm{Id}-\Delta_{-1}\right) \theta\right\|_{\widetilde{L}_{t}^{\varrho} B_{p, r}^{s+\frac{\beta}{\varrho}}} \leq C e^{C U(t)}\left(\left\|\theta_{0}\right\|_{B_{p, r}^{s}}+\|f\|_{L_{t}^{1} B_{p, r}^{s}}\right)
$$


and

$$
\|\theta\|_{\widetilde{L}_{t}^{\varrho} \dot{B}_{p, r}^{s+\frac{\beta}{\varrho}}} \leq C e^{C U(t)}\left(\left\|\theta_{0}\right\|_{\dot{B}_{p, r}^{s}}+\|f\|_{\widetilde{L}_{t}^{\varrho_{1}} \dot{B}_{p, r}^{s+\frac{\beta}{\varrho_{1}}}-\beta}\right),
$$

where $U(t):=\int_{0}^{t}\|\nabla u(\tau)\|_{L^{\infty}} d \tau$.

\section{Modified Riesz transform and commutators}

First we introduce a pseudo-differential operator $\mathcal{R}_{\alpha}$ defined by $\mathcal{R}_{\alpha}:=$ $|D|^{-\alpha} \partial_{1}=|D|^{1-\alpha} \mathcal{R}, 0<\alpha<1$, where $\mathcal{R}:=\frac{\partial_{1}}{|D|}$ is the usual Riesz transform. For convenience we call $\mathcal{R}_{\alpha}$ the modified Riesz transform. We collect some useful properties of this operator as follows.

Proposition 3.1. Let $0<\alpha<1, q \in \mathbb{N}$ and $\mathcal{R}_{\alpha}:=\frac{\partial_{1}}{|D|^{\alpha}}$ be the modified Riesz transform.

(1) Let $\chi \in \mathcal{D}\left(\mathbb{R}^{n}\right)$. Then for every $\left.(p, s) \in[1, \infty] \times\right] \alpha-1, \infty[$,

$$
\left\||D|^{s} \chi\left(2^{-q}|D|\right) \mathcal{R}_{\alpha}\right\|_{\mathcal{L}\left(L^{p}\right)} \lesssim 2^{q(s+1-\alpha)} .
$$

(2) Let $\mathcal{C}$ be a ring. Then there exists $\phi \in \mathcal{S}\left(\mathbb{R}^{n}\right)$ whose spectrum does not meet the origin such that

$$
\mathcal{R}_{\alpha} f=2^{q(n+1-\alpha)} \phi\left(2^{q} \cdot\right) \star f
$$

for every $f$ with Fourier variable supported on $2^{q} \mathcal{C}$.

Remark 3.1. For the point (1), since $|D|^{s} \chi\left(2^{-q}|D|\right) \mathcal{R}_{\alpha}=|D|^{s+1-\alpha} \chi\left(2^{-q}|D|\right) R$, it is thus reduced to the case treated in Proposition 3.1 of [11]. We here note that $|D|^{s} \chi(|D|) R_{\alpha}$ is a convolution operator with kernel $K$ satisfying $|K(x)| \lesssim$ $1 /(1+|x|)^{n+s+1-\alpha}$ for all $x \in \mathbb{R}^{n}$. For the point $(2)$, it can be achieved by a simple cut-off technique.

The following Lemma is useful in dealing with the commutator terms (cf. [11]).

Lemma 3.2. Let $p \in[1, \infty], m \geq p, \bar{m}=\frac{m}{m-1}$ be the dual number and $f, g, h$ belong to the suitable functional spaces. Then,

$$
\begin{gathered}
\|h \star(f g)-f(h \star g)\|_{L^{p}} \leq\|x h\|_{L^{\bar{m}}}\|\nabla f\|_{L^{p}}\|g\|_{L^{m}}, \\
\|h \star(f g)-f(h \star g)\|_{L^{p}} \leq\|x h\|_{L^{1}}\|\nabla f\|_{L^{\infty}}\|g\|_{L^{p}} .
\end{gathered}
$$

The next proposition concerns the crucial commutators involving $R_{\alpha}$.

Proposition 3.3. Let $\alpha \in] 0,1\left[\right.$, u be a smooth divergence-free vector field of $\mathbb{R}^{n}$ and $\theta$ be a smooth scalar function. Then,

(1) for every $s \in] 0, \alpha[$ we have

$$
\left\|\left[\mathcal{R}_{\alpha}, u\right] \theta\right\|_{H^{s}} \lesssim_{s, \alpha}\|\nabla u\|_{L^{2}}\|\theta\|_{B_{\infty, 2}^{s-\alpha}}+\|u\|_{L^{2}}\|\theta\|_{L^{2}} .
$$


In particular, in the 2 dimensional case, if $u:=\Delta^{-1} \nabla^{\perp} \omega$ is given by the

Biot-Savart law and $\omega:=\Gamma+\mathcal{R}_{\alpha} \theta$, we have for every $\left.s \in\right] 0, \alpha[$

$$
\left\|\left[\mathcal{R}_{\alpha}, u\right] \theta\right\|_{H^{s}} \lesssim s, \alpha\|\Gamma\|_{L^{2}}\|\theta\|_{B_{\infty, 2}^{s-\alpha}}+\|\theta\|_{L^{\infty}}\|\theta\|_{H^{s+1-2 \alpha}}+\|u\|_{L^{2}}\|\theta\|_{L^{2}} .
$$

(2) for every $(s, p, r) \in]-1, \alpha[\times[2, \infty] \times[1, \infty]$ we have

$$
\left\|\left[\mathcal{R}_{\alpha}, u \cdot \nabla\right] \theta\right\|_{B_{p, r}^{s}} \lesssim s, \alpha\|\nabla u\|_{L^{p}}\left(\|\theta\|_{B_{\infty, r}^{s+1-\alpha}}+\|\theta\|_{L^{p}}\right) .
$$

Remark 3.2. All the above commutator estimates also hold in the homogeneous framework. For instance, with the same assumption as (3.4) one has

$$
\left\|\left[\mathcal{R}_{\alpha}, u \cdot \nabla\right] \theta\right\|_{\dot{B}_{p, r}^{s}} \lesssim s, \alpha\|\nabla u\|_{L^{p}}\left(\|\theta\|_{\dot{B}_{\infty, r}^{s+1-\alpha}}+\|\theta\|_{L^{p}}\right) .
$$

Proof of Proposition 3.3. (1) We here only treat the special case to get (3.3). First due to Bony's decomposition we split the commutator term into three parts

$$
\begin{aligned}
{\left[\mathcal{R}_{\alpha}, u\right] \theta } & =\sum_{q \in \mathbb{N}}\left[\mathcal{R}_{\alpha}, S_{q-1} u\right] \Delta_{q} \theta+\sum_{q \in \mathbb{N}}\left[\mathcal{R}_{\alpha}, \Delta_{q} u\right] S_{q-1} \theta+\sum_{q \geq-1}\left[\mathcal{R}_{\alpha}, \Delta_{q} u\right] \widetilde{\Delta}_{q} \theta \\
& :=I+I I+I I I .
\end{aligned}
$$

- Estimation of $I$.

Denote $I_{q}:=\left[\mathcal{R}_{\alpha}, S_{q-1} u\right] \Delta_{q} \theta$. Since for each $q \in \mathbb{N}$ the Fourier transform of $I_{q}$ is supported in a ring of size $2^{q}$, from Proposition 3.1-(2) there exists $\phi \in \mathcal{S}\left(\mathbb{R}^{2}\right)$ whose spectrum is away from the origin such that

$$
I_{q}=\left[\phi_{q} \star, S_{q-1} \Delta^{-1} \nabla^{\perp} \Gamma\right] \Delta_{q} \theta+\left[\phi_{q} \star, S_{q-1} \Delta^{-1} \nabla^{\perp} R_{\alpha} \theta\right] \Delta_{q} \theta,
$$

where $\phi_{q}(x):=2^{q(n+1-\alpha)} \phi\left(2^{q} x\right)$. Taking advantage of estimates (3.1) and (3.2), Calderón-Zygmund theorem and Proposition 3.1-(1) we obtain

$$
\begin{aligned}
\left\|I_{q}\right\|_{L^{2}} \leq & \left\|x \phi_{q}\right\|_{L^{1}}\left\|\nabla S_{q-1} \Delta^{-1} \nabla^{\perp} \Gamma\right\|_{L^{2}}\left\|\Delta_{q} \theta\right\|_{L^{\infty}}+ \\
& +\left\|x \phi_{q}\right\|_{L^{1}}\left\|\nabla S_{q-1} \Delta^{-1} \nabla^{\perp} \mathcal{R}_{\alpha} \theta\right\|_{L^{\infty}}\left\|\Delta_{q} \theta\right\|_{L^{2}} \\
\lesssim & 2^{-q \alpha}\|\Gamma\|_{L^{2}}\left\|\Delta_{q} \theta\right\|_{L^{\infty}}+2^{-q \alpha} 2^{q(1-\alpha)}\|\theta\|_{L^{\infty}}\left\|\Delta_{q} \theta\right\|_{L^{2}} .
\end{aligned}
$$

Thus from the support property we directly have

$$
\begin{aligned}
\|I\|_{H^{s}} & \approx\left\|2^{q s}\right\| I_{q}\left\|_{L^{2}}\right\|_{\ell^{2}} \\
& \lesssim\|\Gamma\|_{L^{2}}\|\theta\|_{B_{\infty, 2}^{s-\alpha}}+\|\theta\|_{L^{\infty}}\|\theta\|_{H^{s+1-2 \alpha}} .
\end{aligned}
$$

- Estimation of $I I$.

Denote $I I_{q}:=\left[\mathcal{R}_{\alpha}, \Delta_{q} u\right] S_{q-1} \theta$. As before we have

$$
I I_{q}=\left[\phi_{q} \star, \Delta_{q} \Delta^{-1} \nabla^{\perp} \Gamma\right] S_{q-1} \theta+\left[\phi_{q} \star, \Delta_{q} \Delta^{-1} \nabla^{\perp} \mathcal{R}_{\alpha} \theta\right] S_{q-1} \theta,
$$

and again by using Lemma 3.2 we get

$$
\begin{aligned}
\left\|I I_{q}\right\|_{L^{2}} \leq & \left\|x \phi_{q}\right\|_{L^{1}}\left\|\nabla \Delta_{q} \Delta^{-1} \nabla^{\perp} \Gamma\right\|_{L^{2}}\left\|S_{q-1} \theta\right\|_{L^{\infty}} \\
& +\left\|x \phi_{q}\right\|_{L^{1}}\left\|\nabla \Delta_{q} \Delta^{-1} \nabla^{\perp} \mathcal{R}_{\alpha} \theta\right\|_{L^{2}}\left\|S_{q-1} \theta\right\|_{L^{\infty}} \\
\lesssim & 2^{-q \alpha}\|\Gamma\|_{L^{2}}\left\|S_{q-1} \theta\right\|_{L^{\infty}}+2^{-q \alpha} 2^{q(1-\alpha)}\left\|\Delta_{q} \theta\right\|_{L^{2}}\|\theta\|_{L^{\infty}}
\end{aligned}
$$


Thus discrete Young inequality leads to for every $s<\alpha$

$$
\begin{aligned}
\|I I\|_{H^{s}} & \approx\left\|2^{q s}\right\| I I_{q}\left\|_{L^{2}}\right\|_{\ell^{2}} \\
& \lesssim\|\Gamma\|_{L^{2}}\|\theta\|_{B_{\infty, 2}^{s-\alpha}}+\|\theta\|_{L^{\infty}}\|\theta\|_{H^{s+1-2 \alpha}} .
\end{aligned}
$$

- Estimation of $I I I$.

We divide the term into three parts

$$
\begin{aligned}
I I I & =\sum_{q \geq 0} \mathcal{R}_{\alpha}\left(\Delta_{q} u \widetilde{\Delta}_{q} \theta\right)+\sum_{q \geq 0} \Delta_{q} u\left(\mathcal{R}_{\alpha} \widetilde{\Delta}_{q} \theta\right)+\left[\mathcal{R}_{\alpha}, \Delta_{-1} u\right] \widetilde{\Delta}_{-1} \theta \\
& :=I I I^{1}+I I I^{2}+I I I^{3} .
\end{aligned}
$$

From the following fact that for every $q \geq 0$

$$
\left\|\Delta_{q} u\right\|_{L^{2}} \approx 2^{-q}\left\|\Delta_{q} \omega\right\|_{L^{2}} \lesssim 2^{-q}\left\|\Delta_{q} \Gamma\right\|_{L^{2}}+2^{-q \alpha}\left\|\Delta_{q} \theta\right\|_{L^{2}},
$$

and by a direct computation we have

$$
\begin{aligned}
& 2^{j s}\left\|\Delta_{j} I I I^{1}\right\|_{L^{2}} \\
& \quad \lesssim 2^{j(s+1-\alpha)} \sum_{q \geq j-3, q \geq 0}\left\|\Delta_{q} u\right\|_{L^{2}}\left\|\widetilde{\Delta}_{q} \theta\right\|_{L^{\infty}} \\
& \quad \lesssim 2^{j(s+1-\alpha)} \sum_{q \geq j-3}\left(2^{-q}\left\|\Delta_{q} \Gamma\right\|_{L^{2}}+2^{-q \alpha}\left\|\Delta_{q} \theta\right\|_{L^{2}}\right)\left\|\widetilde{\Delta}_{q} \theta\right\|_{L^{\infty}} \\
& \quad \lesssim \sum_{q \geq j-4} 2^{(j-q)(s+1-\alpha)}\left(2^{q(s-\alpha)}\left\|\Delta_{q} \theta\right\|_{L^{\infty}}\|\Gamma\|_{L^{2}}+2^{q(s+1-2 \alpha)}\left\|\Delta_{q} \theta\right\|_{L^{2}}\|\theta\|_{L^{\infty}}\right) .
\end{aligned}
$$

Thus discrete Young inequality (needing $s+1-\alpha>0$ ) yields

$$
\left\|I I I^{1}\right\|_{H^{s}} \lesssim s, \alpha\|\Gamma\|_{L^{2}}\|\theta\|_{B_{\infty, 2}^{s-\alpha}}+\|\theta\|_{L^{\infty}}\|\theta\|_{H^{s+1-2 \alpha}} .
$$

For $I I I^{2}$, by using Proposition 3.1-(1) and (3.6) we obtain

$$
\begin{aligned}
2^{j s}\left\|\Delta_{j} I I I^{2}\right\|_{L^{2}} & \lesssim 2^{j s} \sum_{q \geq j-3, q \geq 0}\left\|\Delta_{q} u\right\|_{L^{2}}\left\|\mathcal{R}_{\alpha} \widetilde{\Delta}_{q} \theta\right\|_{L^{\infty}} \\
& \lesssim 2^{j s} \sum_{q \geq j-3}\left(2^{-q}\left\|\Delta_{q} \Gamma\right\|_{L^{2}}+2^{-q \alpha}\left\|\Delta_{q} \theta\right\|_{L^{2}}\right) 2^{q(1-\alpha)}\left\|\widetilde{\Delta}_{q} \theta\right\|_{L^{\infty}} \\
& \lesssim \sum_{q \geq j-4} 2^{(j-q) s}\left(2^{q(s-\alpha)}\left\|\Delta_{q} \theta\right\|_{L^{\infty}}\|\Gamma\|_{L^{2}}+2^{q(s+1-2 \alpha)}\right. \\
& \left.\times\left\|\Delta_{q} \theta\right\|_{L^{2}}\|\theta\|_{L^{\infty}}\right) .
\end{aligned}
$$

Using convolution inequality (needing $s>0$ ) again we have

$$
\left\|I I I^{2}\right\|_{H^{s}} \lesssim s, \alpha\|\Gamma\|_{L^{2}}\|\theta\|_{B_{\infty, 2}^{s-\alpha}}+\|\theta\|_{L^{\infty}}\|\theta\|_{H^{s+1-2 \alpha}} .
$$

For $I I I^{3}$, since $\Delta_{j} I I I^{3}=0$ for every $j \geq 3$, then from Bernstein inequality and Calderón-Zygmund theorem we immediately have

$$
\begin{aligned}
\left\|I I I^{3}\right\|_{H^{s}} & \lesssim\left\|\left[\mathcal{R}_{\alpha}, \Delta_{-1} u\right] \widetilde{\Delta}_{-1} \theta\right\|_{L^{2}} \\
& \lesssim\left\|\Delta_{-1} u\right\|_{L^{2}}\left(\left\|\widetilde{\Delta}_{-1} \theta\right\|_{L^{2}}+\left\|\mathcal{R}_{\alpha} \widetilde{\Delta}_{-1} \theta\right\|_{L^{2}}\right) \\
& \lesssim\|u\|_{L^{2}}\|\theta\|_{L^{2}} .
\end{aligned}
$$


This concludes the estimate (3.3).

(2) Once again using Bony's decomposition yields

$$
\begin{aligned}
& {\left[\mathcal{R}_{\alpha}, u \cdot \nabla\right] \theta} \\
& \quad=\sum_{q \in \mathbb{N}}\left[\mathcal{R}_{\alpha}, S_{q-1} u \cdot \nabla\right] \Delta_{q} \theta+\sum_{q \in \mathbb{N}}\left[\mathcal{R}_{\alpha}, \Delta_{q} u \cdot \nabla\right] S_{q-1} \theta+\sum_{q \geq-1}\left[\mathcal{R}_{\alpha}, \Delta_{q} u \cdot \nabla\right] \widetilde{\Delta}_{q} \theta \\
& \quad:=\mathrm{I}+\mathrm{II}+\mathrm{III} .
\end{aligned}
$$

For I, since for every $q \in \mathbb{N}$ the Fourier transform of $S_{q-1} u \Delta_{q} \theta$ is supported in a ring of size $2^{q}$, then from Proposition 3.1-(2) and estimate (3.1), we have for every $j \geq-1$

$$
\begin{aligned}
\left\|\Delta_{j} \mathrm{I}\right\|_{L^{p}} & \lesssim \sum_{|q-j| \leq 4}\left\|\left[\phi_{q} \star, S_{q-1} u \cdot \nabla\right] \Delta_{q} \theta\right\|_{L^{p}} \\
& \lesssim \sum_{|q-j| \leq 4} 2^{-q \alpha}\|\nabla u\|_{L^{p}} 2^{q}\left\|\Delta_{q} \theta\right\|_{L^{\infty}} \\
& \lesssim c_{j} 2^{-j s}\|\nabla u\|_{L^{p}}\|\theta\|_{B_{\infty, r}^{s+1-\alpha}},
\end{aligned}
$$

where $\phi_{q}(x):=2^{q(n+1-\alpha)} \phi\left(2^{q} x\right)$ with $\phi \in \mathcal{S}$ and $\left(c_{j}\right)_{j \geq-1}$ with $\left\|c_{j}\right\|_{\ell^{r}}=1$. Thus we obtain

$$
\|\mathrm{I}\|_{B_{p, r}^{s}} \lesssim\|\nabla u\|_{L^{p}}\|\theta\|_{B_{\infty, r}^{s+1-\alpha}}
$$

For II, as above from a direct calculation we have

$$
\begin{aligned}
\left\|\Delta_{j} \mathrm{II}\right\|_{L^{p}} & \lesssim \sum_{|q-j| \leq 4, q \in \mathbb{N}}\left\|\left[\phi_{q} \star, \Delta_{q} u \cdot \nabla\right] S_{q-1} \theta\right\|_{L^{p}} \\
& \lesssim \sum_{|q-j| \leq 4} 2^{-q \alpha}\|\nabla u\|_{L^{p}}\left\|\nabla S_{q-1} \theta\right\|_{L^{\infty}} \\
& \lesssim\|\nabla u\|_{L^{p}} 2^{-j s} \sum_{-1 \leq q^{\prime} \leq j+2} 2^{\left(q^{\prime}-j\right)(\alpha-s)} 2^{q^{\prime}(s+1-\alpha)}\left\|\Delta_{q^{\prime}} \theta\right\|_{L^{\infty}} .
\end{aligned}
$$

Thus using discrete Young inequality we obtain for every $s<\alpha$

$$
\|\mathrm{II}\|_{B_{p, r}^{s}} \lesssim\|\nabla u\|_{L^{p}}\|\theta\|_{B_{\infty, r}^{s+1-\alpha}}
$$

For III, we further write

$$
\mathrm{III}=\sum_{q \geq 0} \operatorname{div}\left[\mathcal{R}_{\alpha}, \Delta_{q} u\right] \widetilde{\Delta}_{q} \theta+\sum_{1 \leq i \leq n}\left[\partial_{i} \mathcal{R}_{\alpha}, \Delta_{-1} u^{i}\right] \widetilde{\Delta}_{-1} \theta:=\mathrm{III}^{1}+\mathrm{III}^{2} .
$$

For Bernstein inequality and Proposition 3.1-(1), we treat the term III $^{1}$ as follows

$$
\begin{aligned}
& \left\|\Delta_{j} \mathrm{III}^{1}\right\|_{L^{p}} \\
& \leq \sum_{q \geq j-3, q \geq 0}\left\|\Delta_{j} \operatorname{div} \mathcal{R}_{\alpha}\left(\Delta_{q} u \widetilde{\Delta}_{q} \theta\right)\right\|_{L^{p}}+\sum_{q \geq j-3, q \geq 0}\left\|\Delta_{j} \operatorname{div}\left(\Delta_{q} u \mathcal{R}_{\alpha} \widetilde{\Delta}_{q} \theta\right)\right\|_{L^{p}}
\end{aligned}
$$




$$
\begin{aligned}
& \lesssim \sum_{q \geq j-3}\left(2^{j(2-\alpha)}+2^{j} 2^{q(1-\alpha)}\right) 2^{-q}\left\|\Delta_{q} \nabla u\right\|_{L^{p}}\left\|\widetilde{\Delta}_{q} \theta\right\|_{L^{\infty}} \\
& \lesssim\|\nabla u\|_{L^{p}} 2^{-j s} \sum_{q \geq j-4}\left(2^{(j-q)(s+2-\alpha)}+2^{(j-q)(s+1)}\right) 2^{q(s+1-\alpha)}\left\|\Delta_{q} \theta\right\|_{L^{\infty}}
\end{aligned}
$$

Thus we obtain for every $s>-1$

$$
\left\|\mathrm{III}^{1}\right\|_{B_{p, r}^{s}} \lesssim\|\nabla u\|_{L^{p}}\|\theta\|_{B_{\infty, r}^{s+1-\alpha}} .
$$

For the second term, from the spectral property, there exist $\chi^{\prime} \in \mathcal{D}\left(\mathbb{R}^{n}\right)$ such that

$$
\mathrm{III}^{2}=\sum_{1 \leq i \leq n}\left[\partial_{i} R_{\alpha} \chi^{\prime}(D), \Delta_{-1} u^{i}\right] \widetilde{\Delta}_{-1} \theta .
$$

The Proposition 3.1 shows that $\partial_{i} R_{\alpha} \chi^{\prime}(D)$ is a convolution operator with kernel $h^{\prime}$ satisfying

$$
\left|h^{\prime}(x)\right| \leq C(1+|x|)^{-n-2+\alpha}, \quad \forall x \in \mathbb{R}^{n} .
$$

Thus from the fact that $\Delta_{j} \mathrm{III}^{2}=0$ for every $j \geq 3$ and by applying Lemma 3.2 with $m=p \geq 2$, we have

$$
\begin{aligned}
\left\|\mathrm{III}^{2}\right\|_{B_{p, r}^{s}} & \lesssim\left\|\left[h^{\prime} \star, \Delta_{-1} u\right] \widetilde{\Delta}_{-1} \theta\right\|_{L^{p}} \\
& \lesssim\left\|x h^{\prime}\right\|_{L^{\bar{p}}}\left\|\nabla \Delta_{-1} u\right\|_{L^{p}}\left\|\widetilde{\Delta}_{-1} \theta\right\|_{L^{p}} \\
& \lesssim\|\nabla u\|_{L^{p}}\|\theta\|_{L^{p}} .
\end{aligned}
$$

This ends the proof of estimate (3.4).

\section{Proof of Theorem 1.1}

The outline of the proof is as follows: first we give some appropriate a priori estimates, then we prove the uniqueness in a weaker functional framework, and at last we show the existence and treat the continuity-in-time issues.

\subsection{A priori estimates}

Proposition 4.1. Let $(u, \theta)$ be a smooth solution of the BoussinesqNavier-Stokes system (1.1) such that $\left(u^{0}, \theta^{0}\right) \in L^{2} \times L^{2}$. Then for every $m \in[2, \infty]$ and $t \in \mathbb{R}^{+}$

$$
\begin{gathered}
\|\theta\|_{L_{t}^{m} \dot{H} \frac{\beta}{m}} \lesssim\left\|\theta^{0}\right\|_{L^{2}}, \\
\|u\|_{L_{t}^{\infty} L^{2}}^{2}+\|u\|_{L_{t}^{2} \dot{H}^{\frac{\alpha}{2}}}^{2} \leq C_{0}\left(1+t^{2}\right),
\end{gathered}
$$


Besides if $\theta^{0} \in L^{p}$ for some $p \in[1, \infty]$, we further have

$$
\|\theta(t)\|_{L^{p}} \leq\left\|\theta^{0}\right\|_{L^{p}} .
$$

Proof of Proposition 4.1. The $L^{p}$ estimate for $\theta$ is a direct consequence of Proposition 2.2. For the $L^{2}$ estimate of $\theta$, by taking a $L^{2}$-inner product with $\theta$ in the temperature equation we have

$$
\frac{1}{2} \frac{d}{d t}\|\theta(t)\|_{L^{2}}^{2}+\|\theta(t)\|_{\dot{H}^{\frac{\beta}{2}}}^{2}=0 .
$$

Integrating in time leads to

$$
\|\theta(t)\|_{L^{2}}^{2}+2\|\theta\|_{L_{t}^{2} \dot{H}^{\frac{\beta}{2}}}^{2}=\left\|\theta^{0}\right\|_{L^{2}}^{2} .
$$

Thus by interpolation we obtain the desired estimate (4.1). For the $L^{2}$ estimate of $u$, from the standard $L^{2}$ energy estimate, we get

$$
\frac{1}{2} \frac{d}{d t}\|u(t)\|_{L^{2}}^{2}+\|u(t)\|_{\dot{H}^{\frac{\alpha}{2}}}^{2} \leq\|u(t)\|_{L^{2}}\|\theta(t)\|_{L^{2}} .
$$

Thus we obtain

$$
\|u(t)\|_{L^{2}} \leq\left\|u^{0}\right\|_{L^{2}}+\int_{0}^{t}\|\theta(\tau)\|_{L^{2}} \mathrm{~d} \tau \leq\left\|u^{0}\right\|_{L^{2}}+\left\|\theta^{0}\right\|_{L^{2}} t .
$$

Putting this inequality in the previous one yields

$$
\frac{1}{2} \frac{d}{d t}\|u(t)\|_{L^{2}}^{2}+\|u(t)\|_{\dot{H}^{\frac{\alpha}{2}}}^{2} \leq\left\|\theta^{0}\right\|_{L^{2}}\left(\left\|u^{0}\right\|_{L^{2}}+\left\|\theta^{0}\right\|_{L^{2}} t\right) .
$$

Integrating in time again leads to the desired result.

Proposition 4.2. Let $\frac{6-\sqrt{6}}{4}<\alpha<1,1-\alpha<\beta \leq \min \left\{\frac{7+2 \sqrt{6}}{5} \alpha-2, \frac{\alpha(1-\alpha)}{\sqrt{6}-2 \alpha}\right.$, $2-2 \alpha\},(u, \theta)$ be a smooth solution of the Boussinesq-Navier-Stokes system (1.1) such that $\theta^{0} \in H^{1-\alpha} \cap B_{\infty, 1}^{1-\alpha}$ and $u^{0} \in H^{1} \cap \dot{W}^{1, p}$ with $\left.p \in\right] \frac{2}{\alpha+\beta-1}, \infty[$. Then for every $\sigma \in\left[1, \frac{\alpha}{1-\alpha+2 / p}[\right.$

$$
\begin{gathered}
\|u\|_{\widetilde{L}_{t}^{\sigma} B_{\infty, 1}^{1}} \leq \Phi_{3}(t), \\
\|u\|_{\widetilde{L}_{t}^{\infty} H^{1}}+\|u\|_{L_{t}^{\infty} \dot{W}^{1, p}} \leq \Phi_{3}(t), \\
\|\theta\|_{\widetilde{L}_{t}^{\infty}\left(H^{1-\alpha} \cap B_{\infty, 1}^{1-\alpha}\right)}+\|\theta\|_{\widetilde{L}_{t}^{1}\left(H^{1-\alpha+\beta} \cap B_{\infty, 1}^{1-\alpha+\beta}\right)} \leq \Phi_{2}(t) .
\end{gathered}
$$

Moreover if $u^{0}$ also belongs to $\dot{B}_{p, 2}^{1}$, we have $\|u\|_{\widetilde{L}_{t}^{\infty} \dot{B}_{p, 2}^{1}} \leq \Phi_{3}(t)$.

Proof of Proposition 4.2. Denote $\Gamma:=\omega-\mathcal{R}_{\alpha} \theta$. Considering the vorticity equation

$$
\partial_{t} \omega+u \cdot \nabla \omega+|D|^{\alpha} \omega=\partial_{1} \theta
$$

and the acting of $\mathcal{R}_{\alpha}:=|D|^{1-\alpha} \mathcal{R}$ on the temperature equation

$$
\partial_{t} \mathcal{R}_{\alpha} \theta+u \cdot \nabla \mathcal{R}_{\alpha} \theta+|D|^{\beta} \mathcal{R}_{\alpha} \theta=-\left[\mathcal{R}_{\alpha}, u \cdot \nabla\right] \theta,
$$

we directly have

$$
\partial_{t} \Gamma+u \cdot \nabla \Gamma+|D|^{\alpha} \Gamma=\left[\mathcal{R}_{\alpha}, u \cdot \nabla\right] \theta+|D|^{\beta} \mathcal{R}_{\alpha} \theta .
$$


To obtain the key estimate (4.2) with $\sigma=1$, the procedure is as below: we first obtain some "good" estimates on $\Gamma$ through studying the new equation (4.3), and then combining with the estimates of $\theta$ we return to some appropriate estimates on $\omega$ which lead to our target.

- Step 1: Estimation of $\|\Gamma\|_{L_{t}^{\infty} L^{2}}$

From the classical energy method we get for every $s_{1} \in\left[0, \frac{\alpha}{2}\right]$

$$
\begin{aligned}
& \frac{1}{2} \frac{d}{d t}\|\Gamma(t)\|_{L^{2}}^{2}+\|\Gamma(t)\|_{\dot{H}^{\frac{\alpha}{2}}}^{2} \\
& \quad=\int_{\mathrm{R}^{2}} \operatorname{div}\left(\left[\mathcal{R}_{\alpha}, u\right] \theta\right)(t, x) \Gamma(t, x) \mathrm{d} x+\int_{\mathrm{R}^{2}}|D|^{\beta-\alpha} \partial_{1} \theta(t, x) \Gamma(t, x) \mathrm{d} x \\
& \quad \leq\left\|\left[\mathcal{R}_{\alpha}, u\right] \theta(t)\right\|_{\dot{H}^{1-\frac{\alpha}{2}}}\|\Gamma(t)\|_{\dot{H}^{\frac{\alpha}{2}}}+\|\theta(t)\|_{\dot{H}^{1+\beta-\alpha-s_{1}}}\|\Gamma(t)\|_{\dot{H}^{s_{1}}} .
\end{aligned}
$$

Interpolation inequality and Young inequality yield

$$
\begin{aligned}
\|\theta(t)\|_{\dot{H}^{1+\beta-\alpha-s_{1}}}\|\Gamma(t)\|_{\dot{H}^{s_{1}}} & \lesssim\|\theta(t)\|_{\dot{H}^{1+\beta-\alpha-s_{1}}}\|\Gamma(t)\|_{\dot{H}^{\frac{\alpha}{2}}}^{\frac{2 s_{1}}{\alpha}}\|\Gamma(t)\|_{L^{2}}^{1-\frac{2 s_{1}}{\alpha}} \\
& \leq C\|\theta(t)\|_{\dot{H}^{1+\beta-\alpha-s_{1}}}^{2}+C\|\Gamma(t)\|_{L^{2}}^{2}+\frac{1}{4}\|\Gamma(t)\|_{\dot{H}^{\frac{\alpha}{2}}}^{2} .
\end{aligned}
$$

Inserting this inequality into the previous one, and from the continuous embed$\operatorname{ding} H^{1-\frac{\alpha}{2}} \hookrightarrow \dot{H}^{1-\frac{\alpha}{2}}$ and Young inequality again we have

$$
\begin{aligned}
& \frac{d}{d t}\|\Gamma(t)\|_{L^{2}}^{2}+\|\Gamma(t)\|_{\dot{H}^{\frac{\alpha}{2}}}^{2} \\
& \quad \leq 2\left\|\left[\mathcal{R}_{\alpha}, u\right] \theta(t)\right\|_{H^{1-\frac{\alpha}{2}}}^{2}+2 C\|\theta(t)\|_{\dot{H}^{1+\beta-\alpha-s_{1}}}^{2}+2 C\|\Gamma(t)\|_{L^{2}}^{2} .
\end{aligned}
$$

Applying Proposition 3.3 and Proposition 4.1 we have for every $\alpha \in] \frac{2}{3}, 1[$

$$
\begin{aligned}
& \left\|\left[\mathcal{R}_{\alpha}, u\right] \theta(t)\right\|_{H^{1-\frac{\alpha}{2}}} \\
& \quad \lesssim\|\Gamma(t)\|_{L^{2}}\|\theta(t)\|_{B_{\infty, 2}^{1-\frac{3 \alpha}{2}}}+\|\theta(t)\|_{H^{2-\frac{5 \alpha}{2}}}\|\theta(t)\|_{L^{\infty}}+\|u(t)\|_{L^{2}}\|\theta(t)\|_{L^{2}} \\
& \quad \lesssim\|\Gamma(t)\|_{L^{2}}\|\theta(t)\|_{L^{\infty}}+\|\theta(t)\|_{H^{2-\frac{5 \alpha}{2}}}\left\|\theta^{0}\right\|_{L^{\infty}}+(1+t) \\
& \quad \lesssim\|\Gamma(t)\|_{L^{2}}+\|\theta(t)\|_{H^{2-\frac{5 \alpha}{2}}}+(1+t) .
\end{aligned}
$$

Putting the upper estimate in (4.4) we obtain

$$
\frac{d}{d t}\|\Gamma(t)\|_{L^{2}}^{2}+\|\Gamma(t)\|_{\dot{H}^{\frac{\alpha}{2}}}^{2} \lesssim\|\Gamma(t)\|_{L^{2}}^{2}+\|\theta(t)\|_{H^{2-\frac{5 \alpha}{2}}}^{2}+\|\theta(t)\|_{\dot{H}^{1+\beta-\alpha-s_{1}}}^{2}+\left(1+t^{2}\right) .
$$

Gronwall inequality thus leads to

$$
\|\Gamma(t)\|_{L^{2}}^{2}+\int_{0}^{t}\|\Gamma(\tau)\|_{\dot{H}^{\frac{\alpha}{2}}}^{2} \mathrm{~d} \tau \leq C_{1} e^{C_{1} t}\left(1+t^{2}+\|\theta\|_{L_{t}^{2} H^{2-\frac{5 \alpha}{2}}}^{2}+\|\theta\|_{L_{t}^{2} \dot{H}^{1+\beta-\alpha-s_{1}}}^{2}\right) .
$$

If $\frac{3}{4}<\alpha \leq \frac{4}{5}$, we choose $s_{1}=\frac{\alpha}{2}$, and for $1-\alpha<\beta \leq 3 \alpha-2$, then clearly

$$
0 \leq 2-\frac{5 \alpha}{2} \leq \frac{\beta}{2}, \quad 0 \leq 1+\beta-\frac{3 \alpha}{2} \leq \frac{\beta}{2},
$$

by using Proposition 4.1 and interpolation inequality we easily get

$$
\|\theta\|_{L_{t}^{2} H^{2-\frac{5 \alpha}{2}}}^{2}+\|\theta\|_{L_{t}^{2} \dot{H}^{1+\beta-\frac{3 \alpha}{2}}}^{2} \lesssim 1+t .
$$


If $\frac{4}{5}<\alpha<1$, we choose $\left.s_{1}=2-2 \alpha \in\right] 0, \frac{\alpha}{2}[$, and for $1-\alpha<\beta \leq 2-2 \alpha$, then

$$
0 \leq \beta-1+\alpha \leq \frac{\beta}{2}
$$

we also get

$$
\|\theta\|_{L_{t}^{2} H^{2-\frac{5 \alpha}{2}}}^{2}+\|\theta\|_{L_{t}^{2} \dot{H}^{\beta-1+\alpha}}^{2} \lesssim 1+t
$$

Hence for every $\left.\left.(\alpha, \beta) \in \Pi_{2}:=\right] \frac{3}{4}, 1[\times] 1-\alpha, \min \{3 \alpha-2,2-2 \alpha\}\right]$ we have

$$
\|\Gamma(t)\|_{L^{2}}^{2}+\int_{0}^{t}\|\Gamma(\tau)\|_{\dot{H}^{\frac{\alpha}{2}}}^{2} \mathrm{~d} \tau \leq \Phi_{1}(t) .
$$

- Step 2: Estimation of $\|\Gamma\|_{L_{t}^{\infty} L^{\tilde{r}}}$ for every $\widetilde{r} \in[2, r]$ and for some $r \in[2,4[$

Multiplying (4.3) by $|\Gamma|^{r-2} \Gamma$ and integrating in the spatial variable we obtain for every $\left.\left.s_{2}, s_{3} \in\right] 0, \frac{\alpha}{2}\right]\left(s_{3} \leq s_{2}\right.$ and both will be chosen later $)$

$$
\begin{aligned}
& \frac{1}{r} \frac{d}{d t}\|\Gamma(t)\|_{L^{r}}^{r}+\int_{\mathbb{R}^{2}}|D|^{\alpha} \Gamma|\Gamma|^{r-2} \Gamma(t) \mathrm{d} x \\
& \quad \leq \int_{\mathbb{R}^{2}} \operatorname{div}\left[\mathcal{R}_{\alpha}, u\right] \theta|\Gamma|^{r-2} \Gamma(t) \mathrm{d} x+\int_{\mathbb{R}^{2}}|D|^{\beta-\alpha} \partial_{1} \theta|\Gamma|^{r-2} \Gamma(t) \mathrm{d} x \\
& \quad \leq\left\|\left[\mathcal{R}_{\alpha}, u\right] \theta(t)\right\|_{\dot{H}^{1-s_{2}}}\left\||\Gamma|^{r-2} \Gamma(t)\right\|_{\dot{H}^{s_{2}}}+\|\theta(t)\|_{\dot{H}^{1+\beta-\alpha-s_{3}}}\left\||\Gamma|^{r-2} \Gamma(t)\right\|_{\dot{H}^{s_{3}}} .
\end{aligned}
$$

Lemma 3.3 in [14] and continuous embedding $\dot{H}^{\frac{\alpha}{2}} \hookrightarrow L^{\frac{4}{2-\alpha}}$ lead to

$$
\int_{\mathbb{R}^{2}}\left(|D|^{\alpha} \Gamma\right)|\Gamma|^{r-2} \Gamma \mathrm{d} x \gtrsim\left\||\Gamma|^{r / 2}\right\|_{\dot{H}^{\frac{\alpha}{2}}}^{2} \gtrsim\left\||\Gamma|^{r / 2}\right\|_{L^{\frac{4}{2-\alpha}}}^{2}=\|\Gamma\|_{L^{\frac{2 r}{2-\alpha}}}^{r} .
$$

By using Lemma 5.1 in the Appendix we find

$$
\left\||\Gamma|^{r-2} \Gamma\right\|_{\dot{H}^{s_{i}}} \lesssim\|\Gamma\|_{L^{\frac{2 r}{2-\alpha}}}^{r-2}\|\Gamma\|_{\dot{H}^{s_{i}+\left(1-\frac{2}{r}\right)(2-\alpha)}}, \quad i=2,3 .
$$

Collecting the upper estimates we have

$$
\begin{aligned}
& \frac{d}{d t}\|\Gamma(t)\|_{L^{r}}^{r}+\|\Gamma(t)\|_{L^{\frac{2 r}{2-\alpha}}}^{r} \\
& \lesssim\left\|\left[\mathcal{R}_{\alpha}, u\right] \theta(t)\right\|_{\dot{H}^{1-s_{2}}}\|\Gamma(t)\|_{\dot{H}^{s_{2}+\left(1-\frac{2}{r}\right)(2-\alpha)}}\|\Gamma(t)\|_{L^{\frac{2 r}{2-\alpha}}}^{r-2} \\
& \quad+\|\theta(t)\|_{\dot{H}^{1+\beta-\alpha-s_{3}}}\|\Gamma(t)\|_{\dot{H}^{s_{3}}+\left(1-\frac{2}{r}\right)(2-\alpha)}\|\Gamma(t)\|_{L^{\frac{2 r}{2-\alpha}}}^{r-2}
\end{aligned}
$$

Then we choose $s_{2}$ such that $s_{2}+\left(1-\frac{2}{r}\right)(2-\alpha)=\frac{\alpha}{2}$, which calls for $s_{2}=$ $\left.\left.\frac{\alpha}{2}-\left(1-\frac{2}{r}\right)(2-\alpha) \in\right] 0, \frac{\alpha}{2}\right]$, this is plausible if $\left.\alpha \in\right] \frac{4 r-8}{3 r-4}, 1[$ for $r \in[2,4[$. Since $s_{3} \leq s_{2}$, by interpolation we have

$$
\|\Gamma(t)\|_{\dot{H}^{s_{3}}+\left(1-\frac{2}{r}\right)(2-\alpha)} \lesssim\|\Gamma(t)\|_{\dot{H}^{\frac{\alpha}{2}}}^{\delta}\|\Gamma(t)\|_{L^{2}}^{1-\delta} \leq \Phi_{1}(t)\|\Gamma(t)\|_{\dot{H}^{\frac{\alpha}{2}}}^{\delta} .
$$

where $\delta:=\frac{2}{\alpha}\left(s_{3}+\left(1-\frac{2}{r}\right)(2-\alpha)\right)$. Also noticing that if $\left.\alpha \in\right] \frac{6 r-8}{5 r-4}, 1[$, we have $\left.1-s_{2} \in\right] 0, \alpha[$, then from the point (1) of Proposition 3.3 and estimate (4.5) 
we further get

$$
\begin{aligned}
& \left\|\left[\mathcal{R}_{\alpha}, u\right] \theta(t)\right\|_{H^{1-s_{2}}} \\
& \quad \lesssim\|\Gamma(t)\|_{L^{2}}\|\theta(t)\|_{B_{\infty, 2}^{1-s_{2}-\alpha}}+\|\theta(t)\|_{L^{\infty}}\|\theta(t)\|_{H^{2-2 \alpha-s_{2}}}+\|u(t)\|_{L^{2}}\|\theta(t)\|_{L^{2}} \\
& \quad \lesssim\|\Gamma(t)\|_{L^{2}}\|\theta(t)\|_{L^{\infty}}+\left\|\theta^{0}\right\|_{L^{\infty}}\|\theta(t)\|_{H^{2-2 \alpha-s_{2}}}+(1+t) \\
& \quad \lesssim \Phi_{1}(t)+\|\theta(t)\|_{H^{2-2 \alpha-s_{2}}} .
\end{aligned}
$$

Therefore,

$$
\begin{aligned}
\frac{d}{d t}\|\Gamma(t)\|_{L^{r}}^{r}+c\|\Gamma(t)\|_{L^{\frac{2 r}{2-\alpha}}}^{r} \lesssim & \left(\Phi_{1}(t)+\|\theta(t)\|_{H^{2-2 \alpha-s_{2}}}\right)\|\Gamma(t)\|_{L^{\frac{2 r}{2-\alpha}}}^{r-2}\|\Gamma(t)\|_{\dot{H}^{\frac{\alpha}{2}}} \\
& +\Phi_{1}(t)\|\theta(t)\|_{\dot{H}^{1+\beta-\alpha-s_{3}}}\|\Gamma(t)\|_{L^{\frac{2 r}{2-\alpha}}}^{r-2}\|\Gamma(t)\|_{\dot{H}^{\frac{\alpha}{2}}}^{\delta} .
\end{aligned}
$$

According to the following Young inequality

$$
\left.\left.\left|A_{1} A_{2} A_{3}\right| \leq C^{\prime}\left|A_{1}\right|^{\frac{2 r}{4-r \tilde{\delta}}}+C^{\prime \prime}\left|A_{2}\right|^{\frac{2}{\tilde{\delta}}}+\frac{c}{4}\left|A_{3}\right|^{\frac{r}{r-2}}, \quad \forall \widetilde{\delta} \in\right] 0,1\right],
$$

we obtain

$$
\begin{aligned}
& \frac{d}{d t}\|\Gamma(t)\|_{L^{r}}^{r}+\|\Gamma(t)\|_{L^{\frac{2 r}{2-\alpha}}}^{r} \\
\lesssim & \Phi_{1}(t)+\|\theta(t)\|_{H^{2-2 \alpha-s_{2}}}^{\frac{2 r}{4-r}}+\|\Gamma(t)\|_{\dot{H}^{\frac{\alpha}{2}}}^{2}+\left\{\begin{array}{l}
\Phi_{1}(t)\|\theta(t)\|_{\dot{H}^{1+\beta-\alpha-s_{3}}}^{\frac{2 r}{4-\delta r}}, \quad \text { if } \frac{2 r}{4-\delta r} \geq 2 \\
\|\theta(t)\|_{\dot{H}^{1+\beta-\alpha-s_{3}}}^{2}, \quad \text { otherwise. }
\end{array}\right.
\end{aligned}
$$

Integrating in time yields

$$
\begin{aligned}
& \|\Gamma(t)\|_{L^{r}}^{r}+\int_{0}^{t}\|\Gamma(\tau)\|_{L^{\frac{2 r}{2-\alpha}}}^{r} \mathrm{~d} \tau \\
& \lesssim \Phi_{1}(t)+\|\theta\|_{L_{t}^{\frac{2 r}{4-r}} H^{2-2 \alpha-s_{2}}}^{\frac{2 r}{4-r}}+\left\{\begin{array}{l}
\Phi_{1}(t)\|\theta\|^{\frac{2 r}{4-\delta r}}, \quad \text { if } \frac{2 r}{4-\delta r} \geq 2 \\
\|\theta\|_{L_{t}^{2} \dot{H}^{1+\beta-\alpha-s_{3}}}^{2-\delta r} \dot{H}^{1+\beta-\alpha-s_{3}}, \quad \text { otherwise. }
\end{array}\right.
\end{aligned}
$$

Note that we have used (4.5) in the above deduction, thus it means $(\alpha, \beta) \in \Pi_{2}$ at least.

Let $r \in\left[2,4[\right.$. If $\left.\alpha \in] \frac{9 r-12}{8 r-8}, \frac{8 r-8}{7 r-4}\right]$, we choose $s_{3}:=s_{2}=\frac{3 r-4}{2 r} \alpha+\frac{4}{r}-2$, and for $\left.\beta \in] 1-\alpha, \frac{5 r-4}{3 r-4} \alpha-2\right]$, we have

$$
0 \leq 2-2 \alpha-s_{2} \leq \frac{4-r}{2 r} \beta, \quad 0 \leq 1+\beta-\alpha-s_{2} \leq \frac{4-r}{2 r} \beta,
$$

from estimate (4.1) and interpolation inequality we find

$$
\|\theta\|_{L_{t}^{\frac{2 r}{4-r}} \dot{H}^{1+\beta-\alpha-s_{2}}}+\|\theta\|_{L_{t}^{\frac{2 r}{4-r}} H^{2-2 \alpha-s_{2}}} \lesssim 1+t .
$$

If $\alpha \in] \frac{8 r-8}{7 r-4}, 1\left[\right.$, we choose $s_{3}:=2-2 \alpha<s_{2}$, then $\delta=\frac{2}{\alpha}\left(2-2 \alpha+\frac{r-2}{r}(2-\alpha)\right)$ and for $\left.\beta \in] 1-\alpha, \min \left\{\frac{1-\alpha}{\frac{4}{\alpha}\left(1-\frac{1}{r}\right)-2}, 2-2 \alpha\right\}\right]$ we also get

$$
0 \leq \beta-1+\alpha \leq \frac{4-\delta r}{2 r} \beta, \quad 0 \leq \beta-1+\alpha \leq \frac{\beta}{2},
$$

thus

$$
\|\theta\|_{L_{t}^{\frac{2 r}{4-\delta r}} \dot{H}^{1+\beta-\alpha-s_{3}}}+\|\theta\|_{L_{t}^{2} \dot{H}^{1+\beta-\alpha-s_{3}}} \lesssim 1+t .
$$




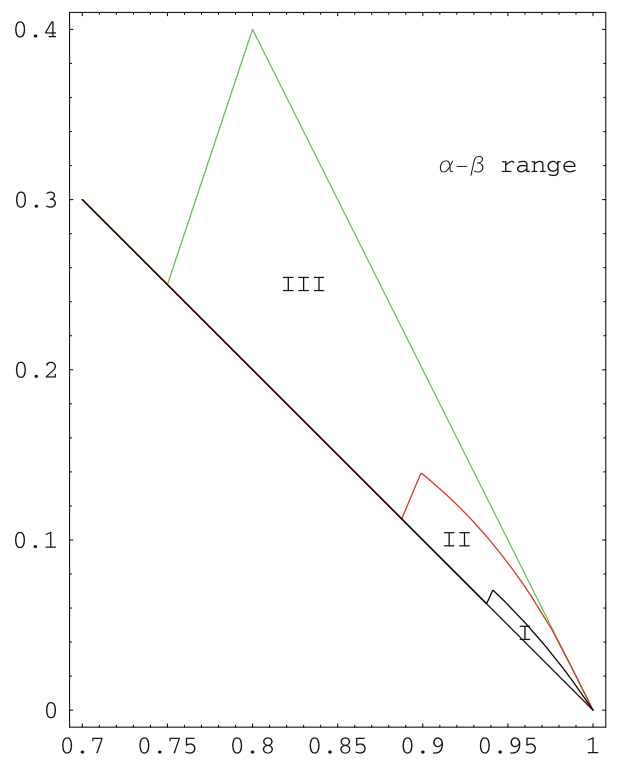

FIgURE 1. III $\supset$ II $\supset$ I with III $\rightarrow \Pi_{2}$, II $\rightarrow \Pi_{\frac{8+2 \sqrt{6}}{5}} \equiv \Pi$, and $\mathrm{I} \rightarrow \Pi_{3}$

Note that as $r \in[2,4[$ increases, the range of $(\alpha, \beta)$ will monotonously shrink (e.g. see Fig. 1). Hence for some $r \in\left[2,4\left[,(\alpha, \beta) \in \Pi_{r}:=\right] \frac{9 r-12}{8 r-8}, 1[\times] 1-\alpha\right.$, $\left.\min \left\{\frac{5 r-4}{3 r-4} \alpha-2, \frac{1-\alpha}{\frac{4}{\alpha}\left(1-\frac{1}{r}\right)-2}, 2-2 \alpha\right\}\right]$, and for every $\widetilde{r} \in[2, r]$ we have for every $t \in \mathbb{R}^{+}$

$$
\|\Gamma(t)\|_{L^{\tilde{r}}}^{\widetilde{r}}+\int_{0}^{t}\|\Gamma(\tau)\|_{L^{2} \frac{2 \tilde{r}}{\widetilde{r}}}^{\widetilde{d}} \mathrm{~d} \tau \Phi_{1}(t) .
$$

- Step 3: Estimation of $\|\omega\|_{L_{t}^{1} L^{\tilde{r}}}$ for every $\widetilde{r} \in[2, r]$ and for some $r \in[2,4[$ Since $\beta>1-\alpha$, there exists a fixed constant $\rho>1$ such that $\frac{\beta}{\rho}>1-\alpha$. From the explicit formula of $\Gamma$ we have for every $\widetilde{r} \in[2, r]$

$$
\begin{aligned}
\|\omega\|_{L_{t}^{1} L^{\tilde{r}}} & \leq\|\Gamma\|_{L_{t}^{1} L^{\tilde{r}}}+\left\|\mathcal{R}_{\alpha} \theta\right\|_{L_{t}^{1} B_{\tilde{r}, 1}^{0}} \\
& \leq \Phi_{1}(t)+t^{1-\frac{1}{\rho}}\left\|\mathcal{R}_{\alpha} \theta\right\|_{\widetilde{L}_{t}^{\rho} B_{\tilde{r}, 1}^{0}} .
\end{aligned}
$$

By a high-low frequency decomposition and a continuous embedding $B_{\tilde{r}, \infty}^{\frac{\beta}{\rho}} \hookrightarrow$ $B_{\widetilde{r}, 1}^{1-\alpha}$ we find

$$
\begin{aligned}
\left\|\mathcal{R}_{\alpha} \theta\right\|_{\tilde{L}_{t}^{\rho} B_{\tilde{r}, 1}^{0}} & \leq\left\|\Delta_{-1} \mathcal{R}_{\alpha} \theta\right\|_{\tilde{L}_{t}^{\rho} B_{\tilde{r}, 1}^{0}}+\left\|\left(I d-\Delta_{-1}\right) \theta\right\|_{\tilde{L}_{t}^{\rho} B_{\tilde{r}, 1}^{1-\alpha}} \\
& \lesssim\left\|\Delta_{-1} \theta\right\|_{L_{t}^{\rho} L^{\tilde{r}}}+\left\|\left(I d-\Delta_{-1}\right) \theta\right\|_{\widetilde{L}_{t}^{\rho} B_{\tilde{r}, \infty}^{\frac{\beta}{\rho}}} \\
& \lesssim t^{\frac{1}{\rho}}\left\|\theta^{0}\right\|_{L^{\tilde{r}}}+\sup _{q \in \mathbb{N}} 2^{q \frac{\beta}{\rho}}\left\|\Delta_{q} \theta\right\|_{L_{t}^{\rho} L^{\tilde{r}}}
\end{aligned}
$$


Inserting this estimate into the previous one and applying Proposition 2.3 we obtain

$$
\|\omega\|_{L_{t}^{1} L^{\tilde{r}}} \leq \Phi_{1}(t)+C t^{1-\frac{1}{\rho}}\|\omega\|_{L_{t}^{1} L^{\tilde{r}}}
$$

where $C$ is an absolute constant depending only on $\widetilde{r}, \rho$ and $\left\|\theta^{0}\right\|_{L^{\infty}}$. If $C t^{1-\frac{1}{\rho}}=\frac{1}{2}$, equivalently, $t=\left(\frac{1}{2 C}\right)^{\rho /(\rho-1)}:=T_{0}$, then for every $t \leq T_{0}$

$$
\|\omega\|_{L_{t}^{1} L^{\tilde{r}}} \leq \Phi_{1}(t) .
$$

Furthermore, if we evolve the system (1.1) from the initial data $\left(u\left(T_{0}\right), \theta\left(T_{0}\right)\right)$, then using the time translation invariance and the fact that $\left\|\theta\left(T_{0}\right)\right\|_{L^{\tilde{r}}} \leq$ $\left\|\theta^{0}\right\|_{L^{\tilde{r}}}$, we have for every $t \leq T_{0}$

$$
\|\omega\|_{L_{\left[T_{0}, T_{0}+t\right]}^{1} L^{\tilde{r}}} \leq \Phi_{1}\left(T_{0}+t\right) .
$$

Iterating like this, we finally get for every $t \in \mathbb{R}^{+}$

$$
\|\omega\|_{L_{t}^{1} L^{\tilde{r}}} \leq \Phi_{1}(t) .
$$

- Step 4: Estimation of $\|\Gamma\|_{\widetilde{L}_{t}^{\sigma} B_{r, 1}^{\frac{2}{r}}}$ for $\sigma \in\left[1, \frac{\alpha}{1-\alpha+2 / r}\left[\right.\right.$ and $r=r_{0}:=\frac{8+2 \sqrt{6}}{5}$

Set $\Gamma_{q}:=\Delta_{q} \Gamma$ for every $q \in \mathbb{N}$. Applying the frequency localization operator $\Delta_{q}$ to the equation (4.3) we get

$$
\begin{aligned}
\partial_{t} \Gamma_{q}+u \cdot \nabla \Gamma_{q}+|D|^{\alpha} \Gamma_{q} & =-\left[\Delta_{q}, u \cdot \nabla\right] \Gamma+\Delta_{q}\left(\left[\mathcal{R}_{\alpha}, u \cdot \nabla\right] \theta\right)+\Delta_{q}|D|^{\beta} \mathcal{R}_{\alpha} \theta \\
& :=f_{q} .
\end{aligned}
$$

Since $\Gamma_{q}$ is real-valued, then after multiplying the upper equation by $\left|\Gamma_{q}\right|^{r-2} \Gamma_{q}$ and integrating in the spatial variable we obtain

$$
\frac{1}{r} \frac{d}{d t}\left\|\Gamma_{q}(t)\right\|_{L^{r}}^{r}+\int_{\mathbb{R}^{2}}\left(|D|^{\alpha} \Gamma_{q}\right)\left|\Gamma_{q}\right|^{r-2} \Gamma \mathrm{d} x \leq\left\|\Gamma_{q}(t)\right\|_{L^{r}}^{r-1}\left\|f_{q}(t)\right\|_{L^{r}} .
$$

Taking advantaging of the following generalized Bernstein inequality (cf. [5])

$$
\int_{\mathbb{R}^{2}}\left(|D|^{\alpha} \Gamma_{q}\right)\left|\Gamma_{q}\right|^{r-2} \Gamma \mathrm{d} x \geq c 2^{q \alpha}\left\|\Gamma_{q}\right\|_{L^{r}}^{r}
$$

with some positive constant $c$ independent of $q$, we have

$$
\frac{1}{r} \frac{d}{d t}\left\|\Gamma_{q}(t)\right\|_{L^{r}}^{r}+c 2^{q \alpha}\left\|\Gamma_{q}(t)\right\|_{L^{r}}^{r} \leq\left\|\Gamma_{q}(t)\right\|_{L^{r}}^{r-1}\left\|f_{q}(t)\right\|_{L^{r}} .
$$

Thus

$$
\left\|\Gamma_{q}(t)\right\|_{L^{r}} \leq e^{-c t 2^{q \alpha}}\left\|\Gamma_{q}^{0}\right\|_{L^{r}}+\int_{0}^{t} e^{-c(t-\tau) 2^{q \alpha}}\left\|f_{q}(\tau)\right\|_{L^{r}} \mathrm{~d} \tau .
$$


By taking the $L^{\sigma}([0, t])$ norm and using the Young inequality we find for every $q \in \mathbb{N}$

$$
\begin{aligned}
2^{q \frac{2}{r}}\left\|\Gamma_{q}\right\|_{L_{t}^{\sigma} L^{r}} \lesssim & 2^{q\left(\frac{2}{r}-\frac{\alpha}{\sigma}\right)}\left\|\Gamma_{q}^{0}\right\|_{L^{r}}+2^{q\left(\frac{2}{r}+1+\beta-\alpha-\frac{\alpha}{\sigma}\right)}\left\|\Delta_{q} \theta\right\|_{L_{t}^{1} L^{r}} \\
& +2^{q\left(\frac{2}{r}+1-\alpha-\frac{\alpha}{\sigma}\right)} \int_{0}^{t} 2^{q(\alpha-1)}\left\|\left[\Delta_{q}, u \cdot \nabla\right] \Gamma(\tau)\right\|_{L^{r}} \mathrm{~d} \tau \\
& +2^{q\left(\frac{2}{r}+1-\alpha-\frac{\alpha}{\sigma}\right)} \int_{0}^{t} 2^{q(\alpha-1)}\left\|\left[\mathcal{R}_{\alpha}, u \cdot \nabla\right] \theta\right\|_{L^{r}} \mathrm{~d} \tau
\end{aligned}
$$

For the second term of the RHS, by using Proposition 2.3, Proposition 4.1 and estimate (4.7) we get for each $q \in \mathbb{N}$

$$
\left\|\Delta_{q} \theta\right\|_{L_{t}^{1} L^{r}} \lesssim 2^{-q \beta}\left(\left\|\theta^{0}\right\|_{L^{r}}+\left\|\theta^{0}\right\|_{L^{\infty}}\|\omega\|_{L_{t}^{1} L^{r}}\right) \leq 2^{-q \beta} \Phi_{1}(t) .
$$

For the fourth term of the RHS of (4.8), we apply estimate (3.4) with $s=\alpha-1$, Proposition 4.1 and estimate (4.7) to obtain

$$
\begin{aligned}
\int_{0}^{t} 2^{q(\alpha-1)}\left\|\left[R_{\alpha}, u \cdot \nabla\right] \theta(\tau)\right\|_{L^{r}} \mathrm{~d} \tau & \lesssim \int_{0}^{t}\|\nabla u(\tau)\|_{L^{r}}\left(\|\theta(\tau)\|_{L^{\infty}}+\|\theta(\tau)\|_{L^{r}}\right) \mathrm{d} \tau \\
& \lesssim\|\omega\|_{L_{t}^{1} L^{r}}\left\|\theta^{0}\right\|_{L^{\infty} \cap L^{r}} \\
& \leq \Phi_{1}(t) .
\end{aligned}
$$

For the third term of the RHS of (4.8), in view of Lemma 5.2-(1) and the specific relationship between $u$ and $\theta$ we infer for every $q \in \mathbb{N}$

$$
\begin{aligned}
2^{q(\alpha-1)}\left\|\left[\Delta_{q}, u \cdot \nabla\right] \Gamma(t)\right\|_{L^{r}} & \lesssim\left(\|\nabla u(t)\|_{B_{r, \infty}^{\alpha-1}}+\|u(t)\|_{L^{2}}\right)\|\Gamma(t)\|_{B_{\infty, \infty}^{0}} \\
& \lesssim\left(\|\Gamma(t)\|_{L^{r}}+\|\theta(t)\|_{L^{r}}+1+t\right)\|\Gamma(t)\|_{B_{r, 1}^{\frac{2}{r}}} \\
& \leq \Phi_{1}(t)\|\Gamma(t)\|_{B_{r, 1}^{\frac{2}{r}}} .
\end{aligned}
$$

To make the series in the sequel summable, we need $\frac{2}{r}+1-\alpha-\frac{\alpha}{\sigma}<0$, that is,

$$
1 \leq \sigma<\frac{\alpha}{1-\alpha+2 / r}, \quad \max \left\{\frac{2+r}{2 r}, \frac{9 r-12}{8 r-8}\right\}<\alpha<1, \quad 2 \leq r<4 .
$$

Since for $r \in\left[2,4\right.$ [ the function $\frac{2+r}{2 r}$ is monotonously decreasing and $\frac{9 r-12}{8 r-8}$ monotonously increasing, to obtain the largest range of $\alpha$, we have to choose $r=r_{0}:=\frac{8+2 \sqrt{6}}{5}$ such that $\frac{2+r}{2 r}=\frac{9 r-12}{8 r-8}=\frac{6-\sqrt{6}}{4}$. This leads to

$$
\left.(\alpha, \beta) \in \Pi=] \frac{6-\sqrt{6}}{4}, 1[\times] 1-\alpha, \min \left\{\frac{7+2 \sqrt{6}}{5} \alpha-2, \frac{\alpha(1-\alpha)}{\sqrt{6}-2 \alpha}, 2-2 \alpha\right\}\right]
$$


Let $Q \in \mathbb{N}$ be a number chosen later. By gathering estimates (4.8)-(4.11) together we find

$$
\begin{aligned}
\|\Gamma\|_{\widetilde{L}_{t}^{\sigma} B_{r, 1}^{\frac{2}{r}}}= & \sum_{q<Q} 2^{q \frac{2}{r}}\|\Gamma\|_{L_{t}^{\sigma} L^{r}}+\sum_{q \geq Q} 2^{q \frac{2}{r}}\|\Gamma\|_{L_{t}^{\sigma} L^{r}} \\
\lesssim & 2^{Q \frac{2}{r}} \Phi_{1}(t)+2^{-Q\left(\frac{\alpha}{\sigma}-\frac{2}{r}\right)}\left\|\Gamma^{0}\right\|_{L^{r}}+2^{-Q\left(\frac{\alpha}{\sigma}+\alpha-1-\frac{2}{r}\right)}\left(\Phi_{1}(t)\right. \\
& +\Phi_{1}(t)\|\Gamma\|_{\left.L_{t}^{1} B_{r, 1}^{\frac{2}{r}}\right)} \\
\leq & \Phi_{1}(t) 2^{Q \frac{2}{r}}+2^{-Q\left(\frac{\alpha}{\sigma}+\alpha-1-\frac{2}{r}\right)} \Phi_{1}(t)\|\Gamma\|_{\widetilde{L}_{t}^{\sigma} B_{r, 1}^{\frac{2}{r}}}
\end{aligned}
$$

We choose $Q$ such that

$$
2^{-Q\left(\frac{\alpha}{\sigma}+\alpha-1-\frac{2}{r}\right)} \Phi_{1}(t) \approx \frac{1}{2}
$$

thus we obtain for every $t \in \mathbb{R}^{+}$

$$
\|\Gamma\|_{\widetilde{L}_{t}^{\sigma} B_{r, 1}^{\frac{2}{r}}} \leq \Phi_{1}(t)
$$

By embedding this immediately leads to

$$
\|\Gamma\|_{\widetilde{L}_{t}^{\sigma} B_{\infty, 1}^{0}} \leq \Phi_{1}(t) .
$$

- Step 5: Estimation of $\|u\|_{L_{t}^{1} B_{\infty, 1}^{1}}$

By virtue of estimate (4.13) with $\sigma=1$ and continuous embedding $B_{\infty, 1}^{0} \cap$ $L^{2} \hookrightarrow L^{\widetilde{p}}$ for all $\widetilde{p} \in[2, p](p \in] \frac{2}{\beta+\alpha-1}, \infty[)$, we get

$$
\begin{aligned}
\|\omega\|_{L_{t}^{1} L^{\tilde{p}}} & \leq\|\Gamma\|_{L_{t}^{1}\left(B_{\infty, 1}^{0} \cap L^{2}\right)}+\left\|\mathcal{R}_{\alpha} \theta\right\|_{L_{t}^{1} B_{\tilde{p}, 1}^{0}} \\
& \leq \Phi_{1}(t)+t^{1-\frac{1}{\rho}}\left\|\mathcal{R}_{\alpha} \theta\right\|_{\widetilde{L}_{t}^{\rho} B_{\tilde{p}, 1}^{0}} .
\end{aligned}
$$

Thus in a similar way as obtaining (4.7), we have for every $\left.\widetilde{p} \in] r_{0}, p\right]$

$$
\|\omega\|_{L_{t}^{1} L \tilde{p}} \leq \Phi_{1}(t) .
$$

From Proposition 2.3, we naturally deduce that

$$
\sup _{q \in \mathbb{N}} 2^{q \beta}\left\|\Delta_{q} \theta\right\|_{L_{t}^{1} L^{\tilde{p}}} \lesssim\left\|\theta^{0}\right\|_{L^{\tilde{p}}}+\left\|\theta^{0}\right\|_{L^{\infty}}\|\omega\|_{L_{t}^{1} L^{\tilde{p}}} \leq \Phi_{1}(t) .
$$

Since $\beta>1-\alpha$ and $p>\frac{2}{\alpha+\beta-1}$, there exists a $\left.\left.p_{\alpha} \in\right] r_{0}, p\right]$ such that $\beta>$ $1-\alpha+\frac{2}{p_{\alpha}}$. Thus

$$
\begin{aligned}
\|\omega\|_{L_{t}^{1} B_{\infty, 1}^{0}} & \leq\|\Gamma\|_{L_{t}^{1} B_{\infty, 1}^{0}}+\left\|R_{\alpha} \theta\right\|_{L_{t}^{1} B_{\infty, 1}^{0}} \\
& \lesssim \Phi_{1}(t)+\left\|\Delta_{-1} R_{\alpha} \theta\right\|_{L_{t}^{1} L^{\infty}}+\sum_{q \in \mathbb{N}}\left\|\Delta_{q} R_{\alpha} \theta\right\|_{L_{t}^{1} L^{\infty}} \\
& \lesssim \Phi_{1}(t)+t\|\theta\|_{L_{t}^{\infty} L^{2}}+\sum_{q \in \mathbb{N}} 2^{q\left(1-\alpha+\frac{2}{p_{\alpha}}-\beta\right)} \sup _{q \in \mathbb{N}} 2^{q \beta}\left\|\Delta_{q} \theta\right\|_{L_{t}^{1} L^{p_{\alpha}}} \\
& \leq \Phi_{1}(t) .
\end{aligned}
$$


This immediately yields

$$
\begin{aligned}
\|u\|_{L_{t}^{1} B_{\infty, 1}^{1}} & \lesssim\left\|\Delta_{-1} u\right\|_{L_{t}^{1} L^{\infty}}+\sum_{q \in \mathbb{N}}\left\|\Delta_{q} \nabla u\right\|_{L_{t}^{1} L^{\infty}} \lesssim\|u\|_{L^{2}}+\|\omega\|_{L_{t}^{1} B_{\infty, 1}^{0}} \\
& \leq \Phi_{1}(t)
\end{aligned}
$$

- Step 6: Estimation of $\|\theta\|_{\widetilde{L}_{t}^{\infty}\left(H^{1-\alpha} \cap B_{\infty, 1}^{1-\alpha}\right)},\|\omega\|_{L_{t}^{\infty} L^{p}}$ and so on.

By Proposition 2.4 and estimate (4.14), we directly obtain

$$
\begin{aligned}
& \|\theta\|_{\widetilde{L}_{t}^{\infty}\left(H^{1-\alpha} \cap B_{\infty, 1}^{1-\alpha}\right)}+\|\theta\|_{\widetilde{L}_{t}^{1}\left(H^{1-\alpha+\beta} \cap B_{\infty, 1}^{1-\alpha+\beta}\right)} \\
& \quad \lesssim\|\theta\|_{\widetilde{L}_{t}^{\infty}\left(H^{1-\alpha} \cap B_{\infty, 1}^{1-\alpha}\right)}+\left\|\left(\operatorname{Id}-\Delta_{-1}\right) \theta\right\|_{\widetilde{L}_{t}^{1}\left(H^{1-\alpha+\beta} \cap B_{\infty, 1}^{1-\alpha+\beta}\right)}+\left\|\Delta_{-1} \theta\right\|_{L_{t}^{1} L^{2} \cap L^{\infty}} \\
& \quad \lesssim e^{C\|\nabla u\|_{L_{t}^{1} L^{\infty}}\left\|\theta^{0}\right\|_{H^{1-\alpha} \cap B_{\infty, 1}^{1-\alpha}}+t\left\|\theta^{0}\right\|_{L^{2} \cap L^{\infty}}} \\
& \quad \lesssim e^{C\|u\|_{L_{t}^{1} B_{\infty, 1}^{1}} \leq \Phi_{2}(t) .}
\end{aligned}
$$

For $p \in] \frac{2}{\alpha+\beta-1}, \infty[$, in light of equation (4.3) and Proposition 2.2 we find

$$
\|\Gamma(t)\|_{L^{p}} \leq\left\|\Gamma^{0}\right\|_{L^{p}}+\int_{0}^{t}\left\|\left[\mathcal{R}_{\alpha}, u \cdot \nabla\right] \theta(\tau)\right\|_{L^{p}} \mathrm{~d} \tau+\int_{0}^{t}\left\||D|^{\beta} \mathcal{R}_{\alpha} \theta(\tau)\right\|_{L^{p}} \mathrm{~d} \tau .
$$

For the first integral of the RHS, using estimate (3.4) with $s=0$ yields

$$
\begin{aligned}
\left\|\left[\mathcal{R}_{\alpha}, u \cdot \nabla\right] \theta(\tau)\right\|_{L^{p}} & \leq\left\|\left[\mathcal{R}_{\alpha}, u \cdot \nabla\right] \theta(\tau)\right\|_{B_{p, 1}^{0}} \\
& \lesssim\|\nabla u(\tau)\|_{L^{p}}\left(\|\theta(\tau)\|_{B_{\infty, 1}^{1-\alpha}}+\|\theta(\tau)\|_{L^{p}}\right) \\
& \leq \Phi_{2}(\tau)\|\omega(\tau)\|_{L^{p}} .
\end{aligned}
$$

For the second integral of the RHS, using Proposition 2.4 again we infer

$$
\begin{aligned}
\int_{0}^{t}\left\||D|^{\beta} \mathcal{R}_{\alpha} \theta(\tau)\right\|_{L^{p}} \mathrm{~d} \tau & \lesssim\left\|\Delta_{-1} \theta\right\|_{L_{t}^{1} L^{p}}+\left\|\left(\mathrm{Id}-\Delta_{-1}\right) \theta\right\|_{L_{t}^{1} B_{p, 1}^{1-\alpha+\beta}} \\
& \lesssim\|\theta\|_{L_{t}^{1} L^{p}}+e^{C\|\nabla u\|_{L_{t}^{1} L^{\infty}}\left\|\theta^{0}\right\|_{B_{p, 1}^{1-\alpha}}} \\
& \leq \Phi_{2}(t) .
\end{aligned}
$$

Hence gathering the upper estimates we have

$$
\begin{aligned}
\|\omega(t)\|_{L^{p}} & \leq\|\Gamma(t)\|_{L^{p}}+\left\|\mathcal{R}_{\alpha} \theta(t)\right\|_{L^{p}} \\
& \leq \Phi_{2}(t)+\int_{0}^{t} \Phi_{2}(\tau)\|\omega(\tau)\|_{L^{p}} \mathrm{~d} \tau .
\end{aligned}
$$

Gronwall inequality ensures

$$
\|\omega(t)\|_{L^{p}} \leq \Phi_{3}(t) .
$$

Taking estimates (4.18) and (4.15) into account, we return to Step 4 and further find for every $\sigma \in\left[1, \frac{\alpha}{1-\alpha+2 / p}[\right.$

$$
\|\Gamma\|_{\widetilde{L}_{t}^{\sigma} B_{p, 1}^{\frac{2}{p}}}+\|u\|_{\widetilde{L}_{t}^{\sigma} B_{\infty, 1}^{1}} \leq \Phi_{3}(t) .
$$


In the proof of the continuity issue below, we also need some a priori bounds on $u$. First, denote $F:=\left[\mathcal{R}_{\alpha}, u \cdot \nabla\right] \theta+|D|^{\beta} \mathcal{R}_{\alpha} \theta$, and estimating as (4.16) and (4.17) we find

$$
\|F\|_{L_{t}^{1} L^{2}} \leq \Phi_{2}(t)\|\omega\|_{L_{t}^{1} L^{2}}+\Phi_{2}(t) \leq \Phi_{2}(t) .
$$

Then, from Proposition 2.4 and estimate (4.15) we obtain

$$
\begin{aligned}
\|\omega\|_{\widetilde{L}_{t}^{\infty} L^{2}} & \leq\|\Gamma\|_{\widetilde{L}_{t}^{\infty} L^{2}}+\left\|\mathcal{R}_{\alpha} \theta\right\|_{\widetilde{L}_{t}^{\infty} L^{2}} \\
& \lesssim e^{C\|\nabla u\|_{L_{t}^{1} L^{\infty}}}\left(\left\|\Gamma^{0}\right\|_{L^{2}}+\|F\|_{L_{t}^{1} L^{2}}\right)+\|\theta\|_{\widetilde{L}_{t}^{\infty} H^{1-\alpha}} \\
& \leq \Phi_{2}(t) .
\end{aligned}
$$

Thus we directly have

$$
\|u\|_{\widetilde{L}_{t}^{\infty} H^{1}} \lesssim\left\|\Delta_{-1} u\right\|_{L_{t}^{\infty} L^{2}}+\|\omega\|_{\widetilde{L}_{t}^{\infty} L^{2}} \leq \Phi_{2}(t) .
$$

If moreover $u^{0} \in \dot{B}_{p, 2}^{1}$, we can also obtain that $u \in \widetilde{L}_{t}^{\infty} \dot{B}_{p, 2}^{1}$. We note that due to $\dot{B}_{p, 2}^{1} \hookrightarrow \dot{W}^{1, p}$ with $\left.p \in\right] \frac{2}{\alpha+\beta-1}, \infty[$, all the Step 1 -Step 5 are satisfied. Then, as above, the key is to estimate $\|\Gamma\|_{\widetilde{L}_{t}^{\infty} \dot{B}_{p, 2}^{0}}$. By virtue of $(2.3)$ with $\varrho_{1}=1$ and (4.14), we get

$$
\|\Gamma\|_{\widetilde{L}_{t}^{\infty} \dot{B}_{p, 2}^{0}} \lesssim \Phi_{2}(t)\left(\left\|\Gamma^{0}\right\|_{\dot{B}_{p, 2}^{0}}+\|F\|_{\widetilde{L}_{t}^{1} \dot{B}_{p, 2}^{0}}\right) .
$$

From $(3.5),(4.15),(4.18)$ and the continuous embedding $B_{\infty, 1}^{1-\alpha} \hookrightarrow \dot{B}_{\infty, 2}^{1-\alpha}$ we find

$$
\left\|\left[\mathcal{R}_{\alpha}, u \cdot \nabla\right] \theta(t)\right\|_{\dot{B}_{p, 2}^{0}} \lesssim\|\nabla u(t)\|_{L^{p}}\left(\|\theta(t)\|_{\dot{B}_{\infty, 2}^{1-\alpha}}+\|\theta(t)\|_{L^{p}}\right) \leq \Phi_{3}(t)
$$

According to (2.3) and the continuous embedding $H^{1-\alpha} \cap B_{\infty, 1}^{1-\alpha} \hookrightarrow \dot{B}_{p, 2}^{1-\alpha}$, we obtain

$$
\left\||D|^{\beta} \mathcal{R}_{\alpha} \theta\right\|_{\widetilde{L}_{t}^{1} \dot{B}_{p, 2}^{0}} \lesssim\|\theta\|_{\widetilde{L}_{t}^{1} \dot{B}_{p, 2}^{1-\alpha+\beta}} \leq \Phi_{2}(t)\left\|\theta^{0}\right\|_{\dot{B}_{p, 2}^{1-\alpha}} \leq \Phi_{2}(t) .
$$

Hence we have

$$
\|\Gamma\|_{\widetilde{L}_{t}^{\infty} \dot{B}_{p, 2}^{0}} \leq \Phi_{2}(t)\left(\left\|\omega^{0}\right\|_{\dot{B}_{p, 2}^{0}}+\left\|\theta^{0}\right\|_{\dot{B}_{p, 2}^{1-\alpha}}+\Phi_{3}(t)\right) \leq \Phi_{3}(t) .
$$

This in turn combining with the relation $\omega=\Gamma+\mathcal{R}_{\alpha} \theta$ and (4.15) leads to

$$
\|u\|_{\widetilde{L}_{t}^{\infty} \dot{B}_{p, 2}^{1}} \lesssim\|\omega\|_{\widetilde{L}_{t}^{\infty} \dot{B}_{p, 2}^{0}} \lesssim\|\Gamma\|_{\widetilde{L}_{t}^{\infty} \dot{B}_{p, 2}^{0}}+\|\theta\|_{\widetilde{L}_{t}^{\infty} \dot{B}_{p, 2}^{1-\alpha}} \leq \Phi_{3}(t) .
$$

This finally ends the proof of Proposition 4.2.

\subsection{Uniqueness}

We shall prove a uniqueness result for the system (1.1) with $(\alpha, \beta) \in \Pi$ in the following space

$$
\mathcal{Z}_{T}:=L_{T}^{\infty} H^{1} \cap L_{T}^{1} B_{\infty, 1}^{1} \times L_{T}^{\infty} L^{2} \cap L_{T}^{1} B_{\infty, 1}^{1-\alpha} .
$$


Let $\left(u_{i}, \theta_{i}\right) \in \mathcal{Z}_{T}$ be two solutions of the system (1.1) with the initial data $\left(u_{i}^{0}, \theta_{i}^{0}\right), i=1,2$. Set $\delta u:=u_{1}-u_{2}, \delta \theta:=\theta_{1}-\theta_{2}$ and $\delta p:=p_{1}-p_{2}$. Then

$$
\begin{aligned}
\partial_{t} \delta u+u_{1} \cdot \nabla \delta u+|D|^{\alpha} \delta u+\nabla \delta p & =-\delta u \cdot \nabla u_{2}+\delta \theta e_{2} \\
\partial_{t} \delta \theta+u_{1} \cdot \nabla \delta \theta+|D|^{\beta} \delta \theta & =-\delta u \cdot \nabla \theta_{2} \\
\left.(\delta u, \delta \theta)\right|_{t=0} & =\left(\delta u^{0}, \delta \theta^{0}\right) .
\end{aligned}
$$

To estimate $\delta u$, by means of Lemma 5.3 (and its remark) in the appendix, we choose $\varrho=1$ for term $-\delta u \cdot \nabla u_{2}$ and $\varrho=\infty$ for term $\delta \theta e_{2}$ to get for every $t \in[0, T]$

$$
\begin{aligned}
\|\delta u(t)\|_{B_{2, \infty}^{0}} \lesssim & e^{C\left\|u_{1}\right\|_{L_{t}^{1} B_{\infty, 1}^{1}}\left(\left\|\delta u^{0}\right\|_{B_{2, \infty}^{0}}\right.}+\int_{0}^{t}\left\|\delta u \cdot \nabla u_{2}(\tau)\right\|_{B_{2, \infty}^{0}} \mathrm{~d} \tau \\
& \left.+(1+t)\|\delta \theta\|_{L_{t}^{\infty} B_{2, \infty}^{-\alpha}}\right) .
\end{aligned}
$$

For the integral term of the RHS, we directly get

$$
\left\|\delta u \cdot \nabla u_{2}\right\|_{B_{2, \infty}^{0}} \lesssim\left\|\delta u \cdot \nabla u_{2}\right\|_{L^{2}} \lesssim\|\delta u\|_{L^{2}}\left\|u_{2}\right\|_{B_{\infty, 1}^{1}} .
$$

Using the logarithmic interpolation inequality stated in Lemma 6.10 of [12] we have

$$
\|\delta u\|_{L^{2}} \lesssim\|\delta u\|_{B_{2, \infty}^{0}} \log \left(e+\frac{1}{\|\delta u\|_{B_{2, \infty}^{0}}}\right) \log \left(e+\|\delta u\|_{H^{1}}\right)
$$

Thus

$$
\left\|\delta u \cdot \nabla u_{2}\right\|_{B_{2, \infty}^{0}} \lesssim\left\|u_{2}\right\|_{B_{\infty, 1}^{1}} \log \left(e+\|\delta u\|_{H^{1}}\right) \mu\left(\|\delta u\|_{B_{2, \infty}^{0}}\right),
$$

where $\mu(x):=x \log \left(e+\frac{1}{x}\right)$. For the last term of the RHS of (4.20), by virtue of Proposition 2.4, we have for every $t \in[0, T]$

$$
\|\delta \theta\|_{L_{t}^{\infty} B_{2, \infty}^{-\alpha}} \lesssim e^{C\left\|u_{1}\right\|_{L_{t}^{1} B_{\infty}^{1}, 1}}\left(\left\|\delta \theta^{0}\right\|_{B_{2, \infty}^{-\alpha}}+\int_{0}^{t}\left\|\delta u \cdot \nabla \theta_{2}(\tau)\right\|_{B_{2, \infty}^{-\alpha}} \mathrm{d} \tau\right) .
$$

Taking advantage of Lemma 5.2 and the logarithmic interpolation inequality again we obtain

$$
\begin{aligned}
\left\|\delta u \cdot \nabla \theta_{2}\right\|_{B_{2, \infty}^{-\alpha}} & \lesssim\|\delta u\|_{L^{2}}\left\|\theta_{2}\right\|_{B_{\infty, 1}^{1-\alpha}} \\
& \lesssim\left\|\theta_{2}\right\|_{B_{\infty, 1}^{1-\alpha}} \log \left(e+\|\delta u\|_{H^{1}}\right) \mu\left(\|\delta u\|_{B_{2, \infty}^{0}}\right) .
\end{aligned}
$$

Denote $Z(t):=\|\delta u\|_{L_{t}^{\infty} B_{2, \infty}^{0}}+\|\delta \theta\|_{L_{t}^{\infty} B_{2, \infty}^{-\alpha}}$. Gathering estimates (4.20)-(4.23) together yields

$$
Z(t) \leq f(t)\left(Z(0)+\int_{0}^{t}\left(\left\|u_{2}(\tau)\right\|_{B_{\infty, 1}^{1}}+\left\|\theta_{2}(\tau)\right\|_{B_{\infty, 1}^{1-\alpha}}\right) \mu(Z(\tau)) \mathrm{d} \tau\right),
$$

where $f(t)$ is an explicit function which continuously and increasingly depends on $\left\|\left(u_{i}, \theta_{i}\right)\right\|_{\mathcal{Z}_{t}}$ and time $t$. Since

$$
\lim _{x \rightarrow 0+} \int_{x}^{1} \frac{1}{\mu(r)} \mathrm{d} r \geq \lim _{x \rightarrow 0+} \log \left(1+\log \frac{1}{x}\right)=\infty
$$


then the classical Osgood lemma (cf. Theorem 5.2.1 in [4]) ensures the uniqueness. Moreover, this lemma also shows some quantified estimates as follows

$$
Z(0) \leq a(T) \Longrightarrow Z(T) \leq b(T)(Z(0))^{\gamma(T)},
$$

where $a, b, \gamma$ are explicit functions depending continuously on $\left\|\left(u_{i}, \theta_{i}\right)\right\|_{\mathcal{Z}_{T}}$ and time $T$.

\subsection{Existence}

First we smooth the data to get the following approximate system

$$
\left\{\begin{array}{l}
\partial_{t} u^{(n)}+u^{(n)} \cdot \nabla u^{(n)}+\nabla p^{(n)}+|D|^{\alpha} u^{(n)}=\theta^{(n)} e_{2} \\
\partial_{t} \theta^{(n)}+u^{(n)} \cdot \nabla \theta^{(n)}+|D|^{\beta} \theta^{(n)}=0 \\
\operatorname{div} u^{(n)}=0 \\
\left.u^{(n)}\right|_{t=0}=S_{n} u^{0},\left.\quad \theta^{(n)}\right|_{t=0}=S_{n} \theta^{0}
\end{array}\right.
$$

Since $S_{n} u^{0}, S_{n} \theta^{0} \in H^{s}$ for every $s \in \mathbb{R}$, from the classical theory of quasi-linear hyperbolic systems, we have the local well-posedness of the approximate system. We also have a blowup criterion as follows: if the quantity $\left\|\nabla u^{n}\right\|_{L_{T}^{1} L^{\infty}}$ is finite, the time $T$ can be continued beyond. Then the a priori estimate (4.2) with $\sigma=1$ ensures that the solution $\left(u^{(n)}, \theta^{(n)}\right)$ is globally defined. Moreover, we also have for $\sigma \in\left[1, \frac{\alpha}{1-\alpha+2 / p}[\right.$,

$$
\begin{array}{r}
\left\|u^{(n)}\right\|_{\widetilde{L}_{T}^{\sigma} B_{\infty, 1}^{1}}+\left\|u^{(n)}\right\|_{\widetilde{L}_{T}^{\infty} H^{1}}+\left\|u^{(n)}\right\|_{L_{T}^{\infty} \dot{W}^{1, p}} \leq \Phi_{3}(T), \\
\left\|\theta^{(n)}\right\|_{\widetilde{L}_{T}^{\infty}\left(H^{1-\alpha} \cap B_{\infty, 1}^{1-\alpha}\right)}+\left\|\theta^{(n)}\right\|_{\widetilde{L}_{t}^{1}\left(H^{1-\alpha+\beta} \cap B_{\infty, 1}^{1-\alpha+\beta}\right)} \leq \Phi_{2}(T) .
\end{array}
$$

Thus there exists $(u, \theta)$ satisfying the above estimates such that $\left(u^{(n)}, \theta^{(n)}\right)$ weakly converges to $(u, \theta)$ up to the extraction of a subsequence. Furthermore, from (4.24), if

$$
d_{n, m}:=\left\|\left(S_{n}-S_{m}\right) u^{0}\right\|_{B_{2, \infty}^{0}}+\left\|\left(S_{n}-S_{m}\right) \theta^{0}\right\|_{B_{2, \infty}^{-\alpha}} \leq a(T)
$$

then we have

$$
\left\|u^{(n)}-u^{(m)}\right\|_{L_{T}^{\infty} B_{2, \infty}^{0}}+\left\|\theta^{(n)}-\theta^{(m)}\right\|_{L_{T}^{\infty} B_{2, \infty}^{-\alpha}} \leq b(T)\left(d_{n, m}\right)^{\gamma(T)} .
$$

This means that $\left(u^{(n)}\right)$ is of Cauchy and thus it converges strongly to $u$ in $L_{T}^{\infty} B_{2, \infty}^{0}$. By interpolation, we obtain the strong convergence of $u^{(n)}$ to $u$ in $L^{2}\left([0, T] \times \mathbb{R}^{2}\right)$. Thus $u^{(n)} \otimes u^{(n)}$ strongly converges in $L^{1}\left([0, T] \times \mathbb{R}^{2}\right)$. But due to that $\theta^{(n)}$ weakly converges to $\theta$ in $L^{2}\left([0, T] \times \mathbb{R}^{2}\right)$, we have $u^{(n)} \theta^{(n)}$ converges weakly to $u \theta$. It then suffices to pass to the limit in (4.25) and we finally get that $(u, \theta)$ is a solution of our original system (1.1).

\subsection{Continuity in time}

We first prove the continuity-in-time of $\theta$ in $B_{\infty, 1}^{1-\alpha}$. Let $\epsilon>0, T>0$, and $J \in \mathbb{N}$ be a number chosen later. Then for every $0 \leq s \leq t \leq T$,

$$
\begin{aligned}
\|\theta(t)-\theta(s)\|_{B_{\infty, 1}^{1-\alpha} \leq} & \sum_{-1 \leq j \leq J} 2^{j(1-\alpha)}\left\|\Delta_{j} \theta(t)-\Delta_{j} \theta(s)\right\|_{L^{\infty}} \\
& +2 \sum_{j>J} 2^{j(1-\alpha)}\left\|\Delta_{j} \theta\right\|_{L_{T}^{\infty} L^{\infty}}
\end{aligned}
$$


Due to $\|\theta\|_{\widetilde{L}_{T}^{\infty} B_{\infty, 1}^{1-\alpha}} \leq \Phi_{2}(T)<\infty$, we can choose $J \in \mathbb{N}$ large enough so that

$$
\sum_{j>J} 2^{j(1-\alpha)}\left\|\Delta_{j} \theta\right\|_{L_{T}^{\infty} L^{\infty}} \lesssim \epsilon
$$

For the first term of the RHS, since for every $-1 \leq j \leq J$,

$$
\begin{aligned}
\left\|\Delta_{j} \theta(t)-\Delta_{j} \theta(s)\right\|_{L^{\infty}} & \leq \int_{s}^{t}\left\|\Delta_{j} \partial_{\tau} \theta(\tau)\right\|_{L^{\infty}} \mathrm{d} \tau \leq(t-s) 2^{j}\left\|\Delta_{j} \partial_{\tau} \theta\right\|_{L_{T}^{\infty} L^{2}} \\
& \leq(t-s) 2^{2 j}\left\|\partial_{\tau} \theta\right\|_{L_{T}^{\infty} B_{2, \infty}^{-1}}
\end{aligned}
$$

and from the equation of $\theta$ and Bony decomposition,

$$
\begin{aligned}
\left\|\partial_{\tau} \theta\right\|_{L_{T}^{\infty} B_{2, \infty}^{-1}} & \leq\|\nabla \cdot(u \theta)\|_{L_{T}^{\infty} B_{2, \infty}^{-1}}+\left\||D|^{\beta} \theta\right\|_{L_{T}^{\infty} B_{2, \infty}^{-1}} \\
& \lesssim\|u\|_{L_{T}^{\infty} L^{2}}\|\theta\|_{L_{T}^{\infty} L^{\infty}}+\|\theta\|_{L_{T}^{\infty} L^{2}} \leq 1+T
\end{aligned}
$$

we obtain

$$
\sum_{-1 \leq j \leq J} 2^{j(1-\alpha)}\left\|\Delta_{j} \theta(t)-\Delta_{j} \theta(s)\right\|_{L^{\infty}} \lesssim(t-s) 2^{J(3-\alpha)}(1+T) .
$$

This indeed ensures the continuity in $B_{\infty, 1}^{1-\alpha}$. To prove $\theta \in \mathcal{C}\left([0, T], H^{1-\alpha}\right)$, as we have $\|\theta\|_{\widetilde{L}_{T}^{\infty} H^{1-\alpha}} \leq \Phi_{2}(T)$ and (4.26), the program is almost the same with the above, and thus we omit it.

Now we turn to the continuity issue of the velocity $u$. First we consider in the topology of $H^{1}$. Since we have $\|u\|_{\widetilde{L}_{T}^{\infty} H^{1}} \leq \Phi_{2}(T)$, similarly as above, it suffices to bound $\left\|\partial_{\tau} u\right\|_{L_{T}^{2} H^{-2+\frac{\alpha}{2}}}$. In fact, from the following formula of $\partial_{\tau} u$

$$
\partial_{\tau} u=-|D|^{\alpha} u-\mathbb{P}(u \cdot \nabla u)-\mathbb{P}\left(\theta e_{2}\right)
$$

with $\mathbb{P}$ the Leray projection operator, we use the Bony decomposition and Sobolev embedding to obtain

$$
\begin{aligned}
\left\|\partial_{\tau} u\right\|_{L_{T}^{2} H^{-2+\frac{\alpha}{2}}} & \lesssim\|u\|_{L_{T}^{2} H^{-2+\frac{3 \alpha}{2}}}+\|u \otimes u\|_{L_{T}^{2} H^{-1+\frac{\alpha}{2}}}+\|\theta\|_{L_{T}^{2} L^{2}} \\
& \lesssim T^{1 / 2}\|u\|_{L_{T}^{\infty} L^{2}}+\|u\|_{L_{T}^{2} H^{\frac{\alpha}{2}}}\|u\|_{L_{T}^{\infty} L^{2}}+T^{1 / 2}\|\theta\|_{L_{T}^{\infty} L^{2}} \\
& \lesssim 1+T^{2}
\end{aligned}
$$

Then, for the continuity-in-time issue in $\dot{W}^{1, p}$ we do not know how to treat it, but alternatively we can prove the continuity in a stronger topology $\dot{B}_{p, 2}^{1}$. In fact, since we have $\|u\|_{\widetilde{L}_{T}^{\infty} \dot{B}_{p, 2}^{1}} \leq \Phi_{3}(T)$, the program is natural. Let $\epsilon>0$ and $J \in \mathbb{N}$ be a fixed number large enough. Then by a direct computation, we have for every $p \in] \frac{2}{\alpha+\beta-1}, \infty[$ and $0 \leq s \leq t \leq T$ 


$$
\begin{aligned}
\|u(t)-u(s)\|_{\dot{B}_{p, 2}^{1}}^{2} & \leq \sum_{|j| \leq J} 2^{2 j}\left\|\dot{\Delta}_{j} u(t)-\dot{\Delta}_{j} u(s)\right\|_{L^{p}}^{2}+\epsilon \\
& \lesssim \sum_{|j| \leq J}\left(2^{j(2-2 / p)} \int_{s}^{t}\left\|\dot{\Delta}_{j} \partial_{\tau} u(\tau, \cdot)\right\|_{L^{2}} \mathrm{~d} \tau\right)^{2}+\epsilon \\
& \lesssim(t-s) \sum_{|j| \leq J} 2^{j(4-4 / p)}\left\|\dot{\Delta}_{j} \partial_{\tau} u\right\|_{L_{T}^{2} L^{2}}^{2}+\epsilon \\
& \lesssim(t-s)\left(J\left\|\Delta_{-1} \partial_{\tau} u\right\|_{L_{T}^{2} L^{2}}^{2}+2^{J\left(8-\frac{4}{p}-\alpha\right)}\left\|\partial_{\tau} u\right\|_{L_{T}^{2} H^{-2+\frac{\alpha}{2}}}^{2}\right)+\epsilon
\end{aligned}
$$

Thus the bound (4.27) definitely ensures the continuity property.

\section{Acknowledgments}

The authors would like to thank Prof. T.Hmidi very much for his helpful discussion and advice. They also want to express their deep gratitude to the anonymous referees for their kind suggestions. The authors were partly supported by the NSF of China (No.10725102).

\section{Appendix: Technical lemmas}

Lemma 5.1. Let $\gamma \in\left[2, \infty[, s \in] 0,1[, \alpha \in] \frac{\gamma-4}{\gamma-2}, 2[\right.$. Then for every smooth function $f$ we have

$$
\left\||f|^{\gamma-2} f\right\|_{\dot{H}^{s}} \lesssim\|f\|_{L^{\frac{2 \gamma}{2-\alpha}}}^{\gamma-2}\|f\|_{\dot{H}^{s+\left(\frac{n}{2}-\frac{n}{\gamma}\right)(2-\alpha)}} .
$$

Proof of Lemma 5.1. This result is a generalization of Lemma 6.9 in [12], and here we sketch the proof. In fact, by Bernstein inequality, it reduces to prove the following stronger result

$$
\left\||f|^{\gamma-2} f\right\|_{\dot{H}^{s}} \lesssim\|f\|_{L^{\frac{2 \gamma}{2-\alpha}}}^{\gamma-2}\|f\|_{\dot{B}_{\tilde{\gamma}, 2}^{s}}
$$

where $\widetilde{\gamma}:=\frac{2 \gamma}{\gamma-(\gamma-2)(2-\alpha)}$. For $\left.s \in\right] 0,1\left[\right.$, we use the characterization of $\dot{H}^{s}$,

$$
\left\||f|^{\gamma-2} f\right\|_{\dot{H}^{s}}^{2} \approx \int_{\mathbb{R}^{n}} \frac{\left\||f|^{\gamma-2} f(x+\cdot)-|f|^{\gamma-2} f(\cdot)\right\|_{L^{2}}^{2}}{|x|^{2 s}} \frac{\mathrm{d} x}{|x|^{n}} .
$$

By using the simple inequality

$$
\left.|| a\right|^{\gamma-2} a-|b|^{\gamma-2} b\left|\lesssim_{\gamma}\right| a-b \mid\left(|a|^{\gamma-2}+|b|^{\gamma-2}\right), \quad \forall a, b \in \mathbb{R},
$$

and Hölder inequality we have for every $\alpha \in] \frac{\gamma-4}{\gamma-2}, 2[$

$$
\begin{aligned}
\left\||f|^{\gamma-2} f(x+\cdot)-|f|^{\gamma-2} f\right\|_{L^{2}} & \lesssim\|f(x+\cdot)-f(\cdot)\|_{L^{\tilde{\gamma}}}\left\||f|^{\gamma-2}\right\|_{L^{\frac{2 \gamma}{(\gamma-2)(2-\alpha)}}} \\
& \lesssim\|f(x+\cdot)-f(\cdot)\|_{L^{\tilde{\gamma}}}\|f\|_{L^{\frac{2 \gamma}{2-\alpha}}}^{\gamma-2} .
\end{aligned}
$$


Hence, from the characterization of homogeneous Besov space again we get

$$
\begin{aligned}
& \left\||f|^{\gamma-2} f\right\|_{\dot{H}^{s}}^{2} \lesssim\|f\|_{L^{2 \gamma} \frac{2 \gamma-4}{2-\alpha}}^{2 \gamma-4} \int_{\mathbb{R}^{n}} \frac{\|f(x+\cdot)-f(\cdot)\|_{L^{\tilde{\gamma}}}^{2}}{|x|^{2 s}} \frac{\mathrm{d} x}{|x|^{n}} \\
& \approx\|f\|_{L^{\frac{2 \gamma}{2-\alpha}}}^{2 \gamma-4}\|f\|_{\dot{B}_{\tilde{\gamma}, 2}^{s}}^{2} .
\end{aligned}
$$

Next we state some useful estimates in Besov framework.

Lemma 5.2. Let $u$ be a smooth divergence-free vector field of $\mathbb{R}^{n}$ and $f$ be a smooth scalar function. Then

(1) for every $\alpha \in] 0,1[$ and $p \in[2, \infty]$

$$
\sup _{q \geq-1} 2^{q(\alpha-1)}\left\|\left[\Delta_{q}, u \cdot \nabla\right] f\right\|_{L^{p}} \lesssim_{\alpha}\left(\|\nabla u\|_{B_{p, \infty}^{\alpha-1}}+\|u\|_{L^{2}}\right)\|f\|_{B_{\infty, \infty}^{0}} .
$$

(2) for every $s \in[-1,0]$

$$
\|u \cdot \nabla f\|_{B_{2, \infty}^{s}} \lesssim\|u\|_{L^{2}}\|f\|_{B_{\infty, 1}^{s+1}} .
$$

Proof of Lemma 5.2. Note that point (2) is just the one in Lemma 6.10 of [12], thus we only need to prove point (1). From Bony's decomposition we have

$$
\begin{gathered}
{\left[\Delta_{q}, u \cdot \nabla\right] f=\sum_{|j-q| \leq 4}\left[\Delta_{q}, S_{j-1} u \cdot \nabla\right] \Delta_{j} f+\sum_{|j-q| \leq 4}\left[\Delta_{q}, \Delta_{j} u \cdot \nabla\right] S_{j-1} f} \\
\quad+\sum_{\substack{j \geq q-3,1 \leq i \leq n\\
}}\left[\Delta_{q} \partial_{i}, \Delta_{j} u^{i}\right] \widetilde{\Delta}_{j} f \\
:=\mathrm{I}_{q}+\mathrm{II}_{q}+\operatorname{III}_{q} .
\end{gathered}
$$

For $\mathrm{I}_{q}$, since $\Delta_{q}:=h_{q}(\cdot) \star=2^{q n} h\left(2^{q} \cdot\right) \star$ with $h \in \mathcal{S}\left(\mathbb{R}^{n}\right)$, then from (3.1) we get for every $\alpha<1$

$$
\begin{aligned}
\left\|\mathrm{I}_{q}\right\|_{L^{p}} \lesssim & \sum_{|j-q| \leq 4}\left\|x h_{q}\right\|_{L^{1}}\left\|\nabla S_{j-1} u\right\|_{L^{p}} 2^{j}\left\|\Delta_{j} f\right\|_{L^{\infty}} \\
\lesssim & \|f\|_{B_{\infty, \infty}^{0}}\|x h\|_{L^{1}} \sum_{|j-q| \leq 4} 2^{j-q} 2^{j(1-\alpha)} \\
& \times \sum_{k \leq j-2} 2^{(j-k)(\alpha-1)} 2^{k(\alpha-1)}\left\|\Delta_{k} \nabla u\right\|_{L^{p}} \\
\lesssim & 2^{q(1-\alpha)}\|\nabla u\|_{B_{p}^{\alpha-\infty}}\|f\|_{B_{\infty, \infty}^{0}},
\end{aligned}
$$

thus

$$
\sup _{q \geq-1} 2^{q(\alpha-1)}\left\|\mathrm{I}_{q}\right\|_{L^{p}} \lesssim\|\nabla u\|_{B_{p, \infty}^{\alpha-1}}\|f\|_{B_{\infty, \infty}^{0}}
$$


For $\mathrm{II}_{q}$, we also use (3.1) to find

$$
\begin{aligned}
\left\|\mathrm{II}_{q}\right\|_{L^{p}} & \lesssim \sum_{|j-q| \leq 4}\left\|x h_{q}\right\|_{L^{1}}\left\|\nabla \Delta_{j} u\right\|_{L^{p}}\left\|S_{j-1} \nabla f\right\|_{L^{\infty}} \\
& \lesssim\|\nabla u\|_{B_{p, \infty}^{\alpha-1}} \sum_{|j-q| \leq 4} 2^{-q} 2^{j(1-\alpha)} \sum_{k \leq j-2} 2^{k}\left\|\Delta_{k} f\right\|_{L^{\infty}} \\
& \lesssim 2^{q(1-\alpha)}\|\nabla u\|_{B_{p, \infty}^{\alpha-1}}\|f\|_{B_{\infty, \infty}^{0}},
\end{aligned}
$$

thus

$$
\sup _{q \geq-1} 2^{q(\alpha-1)}\left\|\mathrm{II}_{q}\right\|_{L^{p}} \lesssim\|\nabla u\|_{B_{p, \infty}^{\alpha-1}}\|f\|_{B_{\infty, \infty}^{0}} .
$$

For $\mathrm{III}_{q}$, we further write

$$
\mathrm{III}_{q}=\sum_{j \geq q-3, j \in \mathbb{N} ; i}\left[\Delta_{q} \partial_{i}, \Delta_{j} u^{i}\right] \widetilde{\Delta}_{j} f+\sum_{1 \leq i \leq n}\left[\Delta_{q} \partial_{i}, \Delta_{-1} u^{i}\right] \widetilde{\Delta}_{-1} f:=\mathrm{III}_{q}^{1}+\mathrm{III}_{q}^{2} .
$$

For the first term, by direct computation we have for every $\alpha>0$

$$
\begin{aligned}
\left\|\operatorname{III}_{q}^{1}\right\|_{L^{p}} & \leq \sum_{j \geq q-3, j \in \mathbb{N} ; i}\left\|\partial_{i} \Delta_{q}\left(\Delta_{j} u^{i} \widetilde{\Delta}_{j} f\right)\right\|_{L^{p}}+\sum_{j \geq q-3, j \in \mathbb{N} ; i}\left\|\Delta_{j} u^{i} \partial_{i} \Delta_{q} \widetilde{\Delta}_{j} f\right\|_{L^{p}} \\
& \lesssim 2^{q(1-\alpha)} \sum_{j \geq q-3, j \in \mathbb{N}} 2^{(q-j) \alpha} 2^{j(\alpha-1)}\left\|\Delta_{j} \nabla u\right\|_{L^{p}}\left\|\widetilde{\Delta}_{j} f\right\|_{L^{\infty}} \\
& \lesssim \alpha 2^{q(1-\alpha)}\|\nabla u\|_{B_{p, \infty}^{\alpha-1}}\|f\|_{B_{\infty, \infty}^{0}},
\end{aligned}
$$

thus

$$
\sup _{q \geq-1} 2^{q(\alpha-1)}\left\|\operatorname{III}_{q}^{1}\right\|_{L^{p}} \lesssim \alpha\|\nabla u\|_{B_{p, \infty}^{\alpha-1}}\|f\|_{B_{\infty, \infty}^{0}} .
$$

For the second term, due to $\mathrm{III}_{q}^{2}=0$ for every $q \geq 3$, we obtain for $p \geq 2$

$$
\sup _{q \geq-1} 2^{q(\alpha-1)}\left\|\operatorname{III}_{q}^{2}\right\|_{L^{p}} \lesssim\left\|\Delta_{-1} u\right\|_{L^{p}}\left\|\widetilde{\Delta}_{-1} f\right\|_{L^{\infty}} \lesssim\|u\|_{L^{2}}\|f\|_{B_{\infty, \infty}^{0}} .
$$

This concludes the proof.

The following estimates on the linearized velocity equation is useful in the proof of the uniqueness part.

Lemma 5.3. Let $s \in]-1,1[, \varrho \in[1, \infty]$ and $v$ be a smooth divergence-free vector field of $\mathbb{R}^{n}$. If $u$ be a smooth solution of the linear system

$$
\partial_{t} u+v \cdot \nabla u+|D|^{\alpha} u+\nabla p=f, \quad \operatorname{div} u=0 .
$$

with $\alpha \in[0,2]$ and $\left.u\right|_{t=0}=u^{0}$, then for every $t \in \mathbb{R}^{+}$we have

$$
\|u\|_{L_{t}^{\infty} B_{2, \infty}^{s}} \leq C e^{C V(t)}\left(\left\|u^{0}\right\|_{B_{2, \infty}^{s}}+\left(1+t^{1-\frac{1}{\varrho}}\right)\|f\|_{\widetilde{L}_{t}^{\varrho} B_{2, \infty}^{s+\frac{\alpha}{\varrho}-\alpha}}\right),
$$

where $V(t):=\int_{0}^{t}\|\nabla v(\tau)\|_{L^{\infty}} d \tau$.

Remark 5.1. The proof can be done in a similar way as obtaining Proposition 4.3 in [12]. We also note that if $f=f_{1}+f_{2}$, one can choose different $\varrho_{1}, \varrho_{2}$ to suit $f_{1}, f_{2}$ respectively. 


\section{References}

[1] Abidi, H., Hmidi, T.: On the global wellposedness of the critical quasigeostrophic equation. SIAM J. Math. Anal. 40, 167-185 (2008)

[2] Beale, J., Kato, T., Majda, A.: Remarks on the breakdown of smooth solutions for the 3-D Euler equations. Commun. Math. Phys. 94, 61-66 (1984)

[3] Chae, D.: Global regularity for the 2-D Boussinesq equations with partial viscous terms. Adv. Math. 203(2), 497-513 (2006)

[4] Chemin, J-Y.: Perfect Incompressible Fluids. Clarendon Press, Oxford (1998)

[5] Chen, Q., Miao, C., Zhang, Z.: A new Bernstein's inequality and the 2D dissipative quasi-geostrophic equation. Commun. Math. Phys. 271, 821-838 (2007)

[6] Córdoba, A., Córdoba, D.: A maximum principle applied to the quasigeostrophic equations. Commun. Math. Phys. 249, 511-528 (2004)

[7] Danchin, R.: Estimates in Besov spaces for transport and transportdiffusion equations with almost Lipschitz coefficients. Rev. Mat. Iberoamericana 21(3), 861-886 (2005)

[8] Danchin, R., Paicu, M.: Le théorème de Leray et le théorèmede FujitaKato pour le système de Boussinesq partiellement visqueux. Bull. Soc. Math. France 136(2), 261-309 (2008)

[9] Hmidi, T., Keraani, S.: On the global well-posedness of the two-dimensional Boussinesq system with a zero diffusivity. Adv. Differ. Equ. 12(4), 461-480 (2007)

[10] Hmidi, T., Keraani, S.: On the global well-posedness of the two-dimensional Boussinesq system with a zero viscosity. Indiana Univ. Math. J. 58(4), 1591-1618 (2009)

[11] Hmidi, T., Keraani, S., Rousset, F.: Global well-posedness for Euler-Boussinesq system with critical dissipation. Commun. Partial Differ. Equ. 36(3), 420-445 (2011)

[12] Hmidi, T., Keraani, S., Rousset, F.: Global well-posedness for a BoussinesqNavier-Stokes system with critical dissipation. J. Differ. Equ. 249(9), 2147-2174 (2010)

[13] Hou, T.Y., Li, C.: Global well-posedness of the viscous Boussinesq equations. Discrete Contin. Dyn. Syst. A 12(1), 1-12 (2005)

[14] Ju, N.: The maximal principle and the global attrator for the dissipative 2D quasi-geostrophic equations. Commun. Math. Phys. 255, 161-181 (2005)

[15] Miao, C., Wu, G.: Global well-posedness of the critical Burgers equation in critical Besov spaces. J. Differ. Equ. 247, 1673-1693 (2009)

[16] Pedlosky, J.: Geophysical Fluid Dynamics. Springer, New York (1987) 
[17] Xu, X.: Global regularity of solutions of $2 \mathrm{D}$ Boussinesq equations with fractional diffusion. Nonlinear Anal. 72, 677-681 (2010)

[18] Xue, L.: Global well-posedness for the two-dimensional Boussinesq systems with partial subcritical viscosity. (2011, submitted)

Changxing Miao

Institute of Applied Physics and Computational Mathematics

P.O. Box 8009

Beijing 100088

People's Republic of China

e-mail: miao_changxing@iapcm.ac.cn

Liutang Xue

The Graduate School of China Academy of Engineering Physics

P.O. Box 2101

Beijing 100088

People's Republic of China

e-mail: xue_lt@163.com

Received: 3 April 2010.

Accepted: 20 April 2011. 